\title{
Physical Mechanisms of the Rogue Wave Phenomenon
}

\author{
Christian Kharif $^{1)}$ and Efim Pelinovsky ${ }^{2)}$
}

\begin{abstract}
${ }^{1)}$ Institut de Recherche sur les Phenomenes Hors Equilibre (IRPHE), Technopole de Chateau-Gombert, 49 rue Joliot Curie BP 146, 13384 MARSEILLE cedex 13, France, E-mail: kharif@irphe.univ-mrs.fr

${ }^{2)}$ Laboratory of Hydrophysics and Nonlinear Acoustics, Institute of Applied Physics, 46 Uljanov Street, Nizhny Novgorod, 603950 Russia, E-mail: enpeli@hydro.appl.sci-nnov.ru
\end{abstract}

\begin{abstract}
:
A review of physical mechanisms of the rogue wave phenomenon is given. The data of marine observations as well as laboratory experiments are briefly discussed. They demonstrate that freak waves may appear in deep and shallow waters. Simple statistical analysis of the rogue wave probability based on the assumption of a Gaussian wave field is reproduced. In the context of water wave theories the probabilistic approach shows that numerical simulations of freak waves should be made for very long times on large spatial domains and large number of realizations. As linear models of freak waves the following mechanisms are considered: dispersion enhancement of transient wave groups, geometrical focusing in basins of variable depth, and wave-current interaction. Taking into account nonlinearity of the water waves, these mechanisms remain valid but should be modified. Also, the influence of the nonlinear modulational instability (BenjaminFeir instability) on the rogue wave occurence is discussed. Specific numerical simulations were performed in the framework of classical nonlinear evolution equations: the nonlinear Schrödinger equation, the Davey - Stewartson system, the Korteweg - de Vries equation, the Kadomtsev - Petviashvili equation, the Zakharov equation, and the fully nonlinear potential equations. Their results show the main features of the physical mechanisms of rogue wave phenomenon.
\end{abstract}

May 5, 2003

Correspondence to: Prof. Christian Kharif (E-mail: kharif@irphe.univ-mrs.fr) 


\section{Introduction}

Freak, rogue, or giant waves correspond to large-amplitude waves surprisingly appearing on the sea surface ("wave from nowhere"). Such waves can be accompanied by deep troughs (holes), which occur before and/or after the largest crest. As it is pointed by Lawton (2001) the freak waves have been part of marine folklore for centuries. Seafarers speak of "walls of water", or of "holes in the sea", or of several successive high waves ("three sisters"), which appear without warning in otherwise benign conditions. But since 70 s of last century, oceanographers have started to believe them. Observations gathered by the oil and shipping industries suggest there really is something out a true monster of the deep that devours ships and sailors without mercy or warning. There are several definitions for such surprising huge waves. Very often the term "extreme waves" is used to specify the tail of some typical statistical distribution of wave heights (generally a Rayleigh distribution), meanwhile the term "freak waves" describes the largeamplitude waves occurring more often than would be expected from the background probability distribution. Recently, Haver \& Andersen (2000) put the question, what is a freak wave: rare realization of a typical statistics or typical realization of a rare population. Sometimes, the definition of the freak waves includes that such waves are too high, too asymmetric and too steep. More popular now is the amplitude criterion of freak waves: its height should exceed the significant wave height in 2-2.2 times. Due to the rare character of the rogue waves their prediction based on data analysis with use of statistical methods is not too productive. During last 30 years the various physical models of the rogue wave phenomenon have been intensively developed and many laboratory experiments conducted. The main goal of these investigations is to understand the physics of the huge wave appearance and its relation to environmental conditions (wind and atmospheric pressure, bathymetry and current field) and to provide the "design" of freak wave needed for engineering purposes. A great progress is achieved in the understanding of the physical mechanisms of the rogue wave phenomenon during the last five years and the paper contains the review of developed models of freak waves.

The paper is organized as following. Data of freak wave observations are collected in section 2 . We demonstrate that freak waves appear in basins of arbitrary depth (in deep, as well as in shallow water) with/without strong current. Freak waves may have solitary-like shape or correspond to a group of several waves. Freak waves have quasi-plane wave fronts, and therefore they can be sought as 2D or anisotropic 3D waves. Briefly, the probability of the rogue wave occurrence is discussed in section 3 in the framework of the Rayleigh statistics. This analysis shows that a large body of numerical simulations should be performed to verify the theoretical scenarios and have reliable prediction of freak waves. Linear mechanisms of the rogue wave 
phenomenon are investigated in section 4. Assuming that the wind wave field in the linear theory can be considered as the sum of a very large number of independent monochromatic waves with different frequencies and directions, a freak wave may appear in the process of spatial wave focusing (geometrical focusing), and spatio-temporal focusing (dispersion enhancement). Also, wave - current interactions can be at the origin of large wave events. Very briefly, the role of atmospheric forcing in the rogue wave phenomenon is pointed out; very few papers consider this aspect. Because the freak wave is a large-amplitude steep wave, nonlinearity plays an important role in the formation of huge waves. These processes are discussed in section 5. Nonlinearity modifies the focusing mechanisms due to the optimal phase relations between spectral components, but does not destroy them. Focusing mechanisms are robust with respect to random wave components. A new mechanism of freak wave formation, suggested in the framework of nonlinear theory only, is the modulational instability (Benjamin - Feir instability). This mechanism is effective for waves in basins of arbitrary depth but not for shallow water. To compare with focusing mechanisms, the modulational instability occurs when the random wave components are weak. All the processes mentioned above are investigated in the framework of weakly nonlinear models like the nonlinear Schrödinger equation, the Davey-Stewartson system, the Korteweg-de Vries equation, and the Kadomtsev-Petviashvili equation. Recently the freak wave phenomenon has been considered by using higher-order nonlinear and dispersive models (like the Zakharov and Dysthe equations) and the fully nonlinear potential equations; the results are presented in section 5. A nonlinear model of wave-current interaction in the vicinity of the blocking point is briefly presented. Some solutions, illustrated by envelope soliton penetration and reflection on opposite current, are given. In conclusion, perspectives in the study of rogue wave phenomenon are discussed towards the assessment of potential design of freak waves.

\section{Freak Wave Observations}

Recently, a large collection of freak wave observations from ships was given in the New Scientist Magazine (Lawton, 2001). In particular, twenty-two super-carriers were lost due to collisions with rogue waves for 1969-1994 in the Pacific and Atlantic causing 525 fatalities, see Figure 2.1. At least, the twelve events of the ship collisions with freak waves were recorded after 1952 in the Indian Ocean, near the Agulhas Current, coast off South Africa (Lavrenov, 1998). Probably, the last event occurred in shallow water $4^{\text {th }}$ November 2000 with the NOAA vessel; the text below is an event description reproduced from Graham (2000).

"At 11:30 a.m. last Saturday morning (November 4, 2000), the 56-foot research vessel R/V Ballena capsized in a rogue wave south of Point Arguello, California. The Channel Islands National Marine Sanctuary's research vessel was engaged in a routine side-scan sonar survey for the U. S. Geological 
Survey of the seafloor along the 30-foot-depth contour approximately 1/4 nautical mile from the shore. The crew of the R/V Ballena, all of whom survived, consisted of the captain, NOAA Corps officer LCdr. Mark Pickett, USGS research scientist Dr. Guy Cochrane, and USGS research assistant, Mike Boyle. According to National Oceanic \& Atmospheric Administration spokesman Matthew Stout, the weather was good, with clear skies and glassy swells. The forecasted swell was 7 feet and the actual swell appeared to be 5-7 feet. At approximately 11:30 a.m., Pickett and Boyle said they observed a 15-foot swell begin to break 100 feet from the vessel. The wave crested and broke above the vessel, caught the Ballena broadside, and quickly overturned her. All crewmembers were able to escape the overturned vessel and deploy the vessel's liferaft. The crew attempted to paddle to the shore, but realized the possibility of navigating the raft safely to shore was unlikely due to strong near-shore currents. The crew abandoned the liferaft approximately 150 feet from shore and attempted to swim to safety. After reaching shore, Pickett swam back out first to assist Boyle to safety and again to assist Cochrane safely to shore. The crew climbed the rocky cliffs along the shore. The R/V Ballena is a total loss."

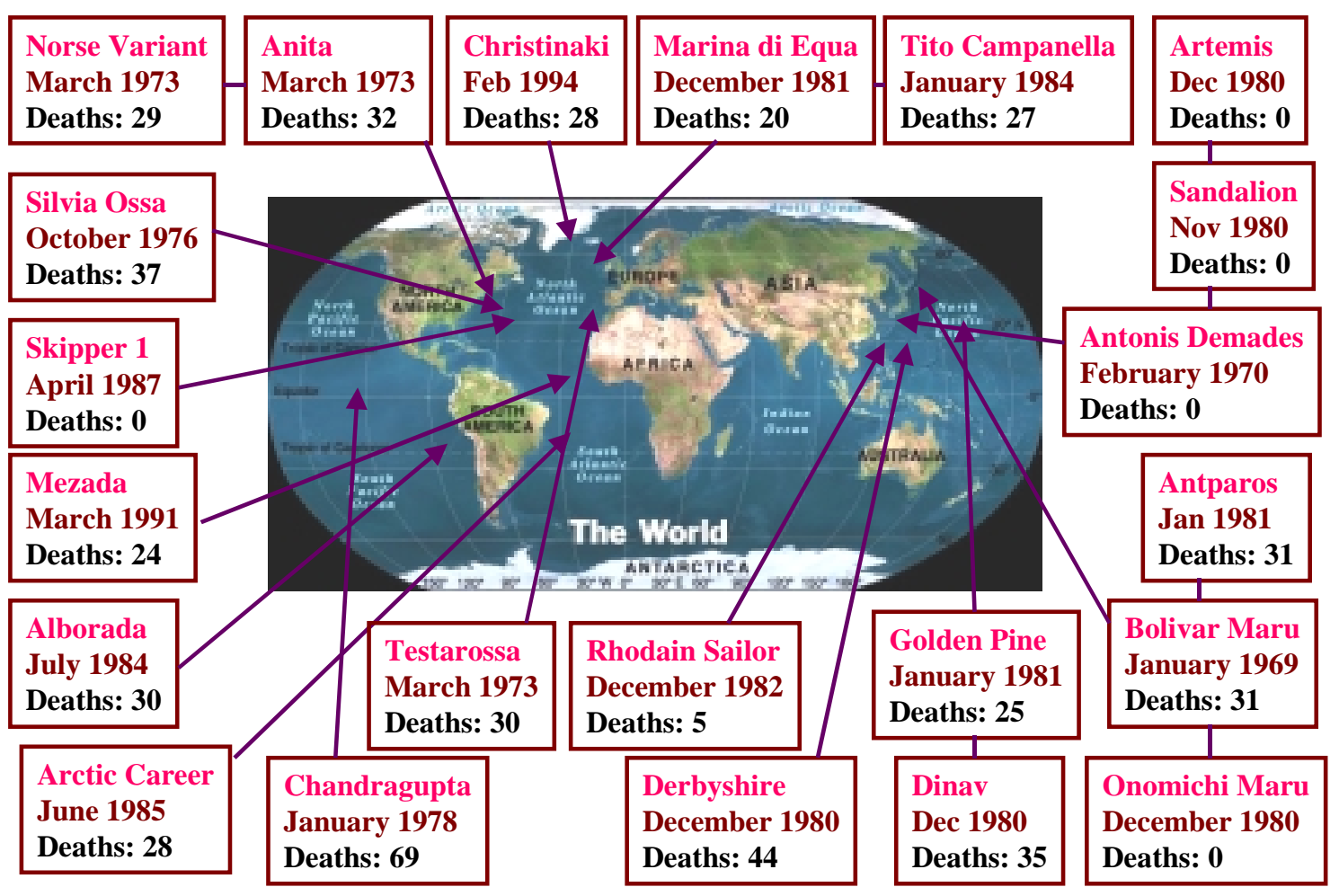

Figure 2.1. Statistics of the super-carrier collision with rogue waves for 1968-1994

Various photos of freak wave are displayed in Figure 2.2 (Olagnon, 2000). The description of the conditions when one of the photos (left upper) was taken is given below (Chase, web).

"A substantial gale was moving across Long Island, sending a very long swell down our way, meeting the Gulf Stream. We saw several rogue waves during the late morning on the horizon, but thought they were whales jumping. It was actually a nice day with light breezes and no significant sea. Only the very long swell, of about 15 feet high and probably 600 to 1000 feet long. This one hit us at the change of the watch at about noon. The photographer was an engineer, and this was the last photo on his roll of film. We 
were on the wing of the bridge, with a height of eye of 56 feet, and this wave broke over our heads. This shot was taken as we were diving down off the face of the second of a set of three waves, so the ship just kept falling into the trough, which just kept opening up under us. It bent the foremast (shown) back about 20 degrees, tore the foreword firefighting station (also shown) off the deck (rails, monitor, platform and all) and threw it against the face of the house. It also bent all the catwalks back severely. Later that night, about 19:30, another wave hit the after house, hitting the stack and sending solid water down into the engine room through the forced draft blower intakes."
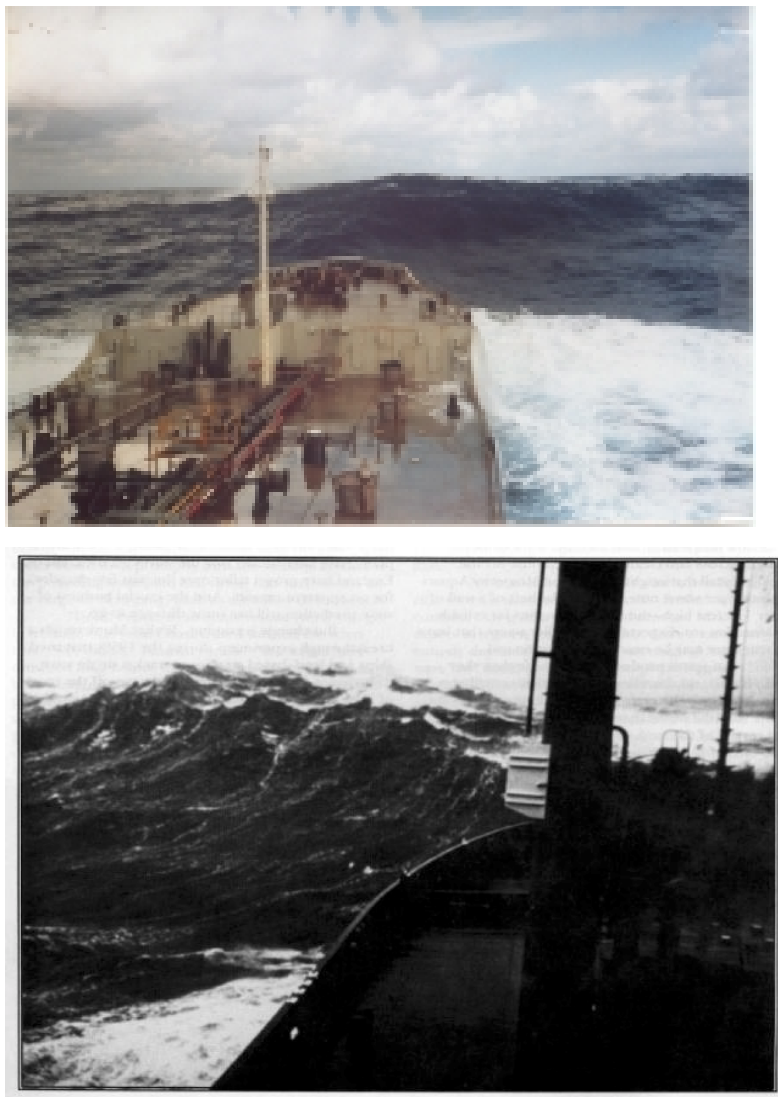
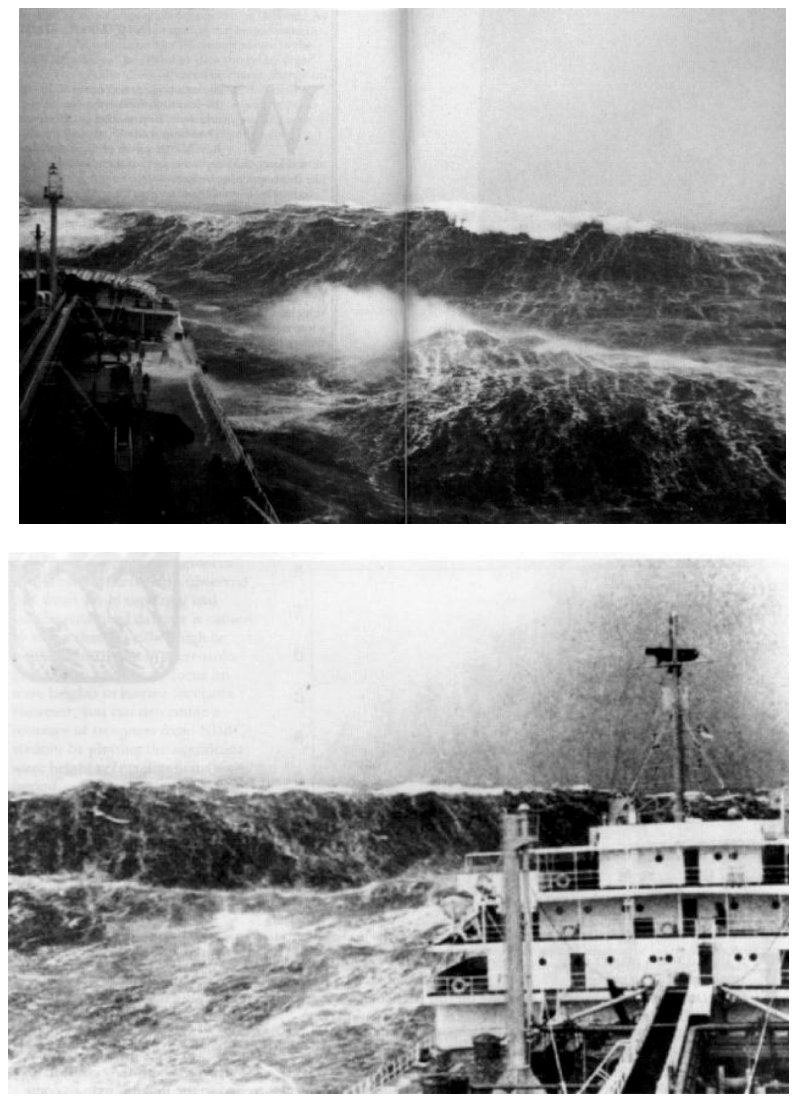

Figure 2.2. Various photos of rogue waves

These photos and descriptions show the main features of the freak wave phenomenon: the rapid appearance of large amplitude solitary pulses or a group of large amplitude waves on the almost still water in shallow as well as in deep water. They highlight also the nonlinear character of the rogue wave shapes: steep front or crest beard, and also two- and three-dimensional aspects of the wave field.

The instrumental data of the freak wave registration are obtained for different oil platforms. Figure 2.3 shows the famous "New year wave" of $26 \mathrm{~m}$ height recorded at "Draupner" (Statoil operated jacket platform, Norway) in the North Sea $1^{\text {st }}$ January 1995 (Haver \& Andersen, 2000). The water depth, $h$, is $70 \mathrm{~m}$, the characteristic period of freak wave is $12 \mathrm{~s}$; so, the wavelength is about $220 \mathrm{~m}$ according to the linear dispersion relation. The important parameter of dispersion, $k h$, is $k h \sim 2$ and this means that the observed freak wave can be considered as a wave propagating in finite depth. Nonlinearity of this wave can be characterized by the steepness, $k a(k$ 
is the wave number and a is the wave amplitude), and it is 0.37 . Alternative nonlinear parameter, $a / h$, is about 0.2 . It should be noted that nonlinearity of the freak wave is very high. Sand et al. (1990) have collected data of freak wave observations in the North Sea (depth 20-40 m) for 1969-1985. Maximum ratio of the freak wave height, $H_{f}$, to the significant wave height, $H_{s}$, reached 3 (Hanstholm, Danish Sector, depth $20 \mathrm{~m}, H_{s}=2 \mathrm{~m}, H_{f}=6 \mathrm{~m}$ ). Such an event can be classified as a freak wave phenomenon in shallow water. Recently, Mori et al. (2002) published an analysis of freak wave observations (at least 14 times with the height exceeding $10 \mathrm{~m}$ ) in the Japan Sea (Yura Harbor, 43 m depth) during 1986-1990. Maximum ratio, $H_{f} / H_{s}$ reached 2.67.

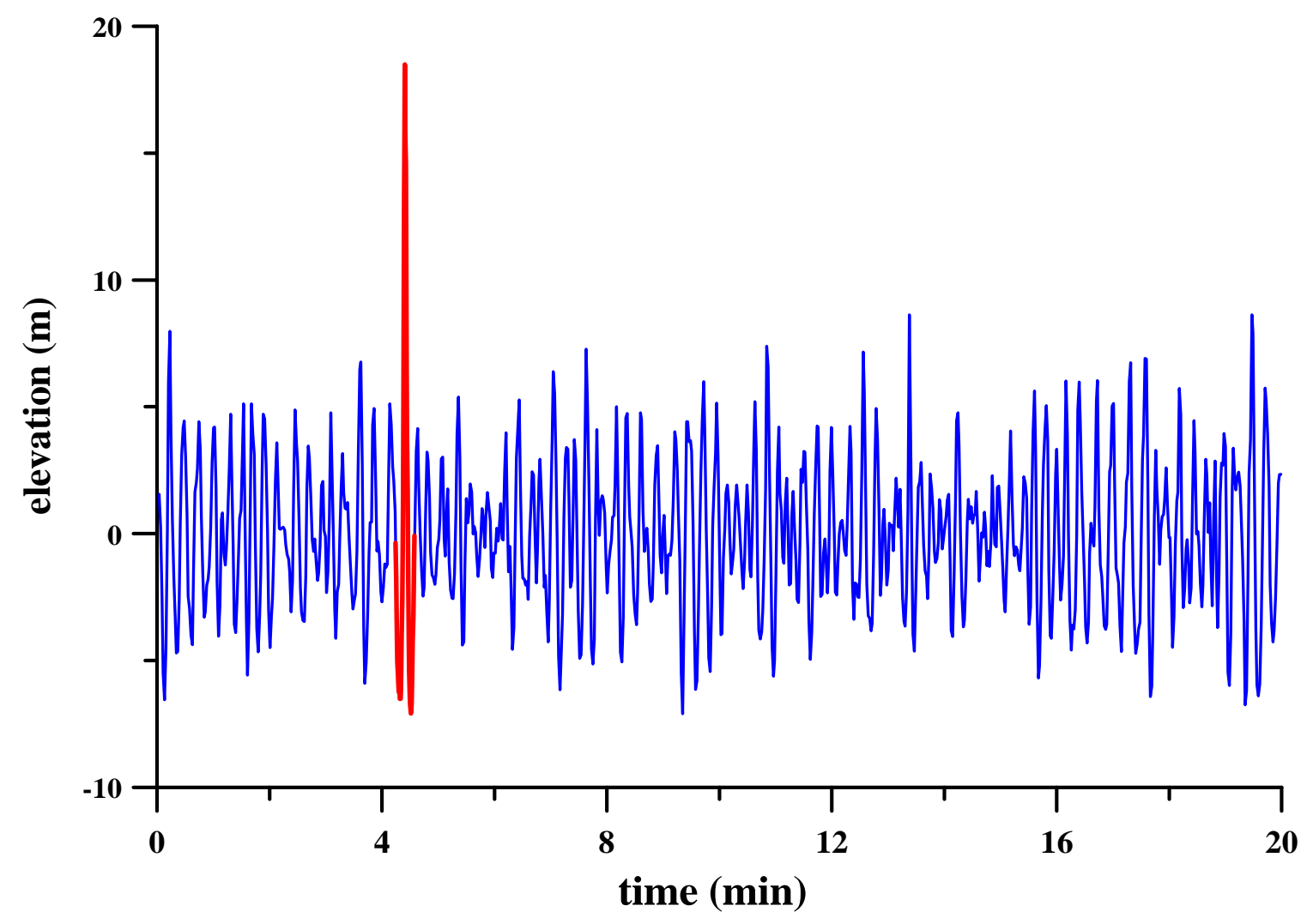

Figure 2.3. Time record of the "New Year wave" in the North Sea

All data given above demonstrate that freak waves can appear in basins of arbitrary depth (deep, intermediate, shallow) with/without strong current. Their main features are: rare and short-lived character of this phenomenon, solitary-like shape or a group of the several waves, high nonlinearity, and quasi-plane wave fronts.

Laboratory experiments provide also a wide variety in the forms of the giant waves (Baldock and Swan, 1996; Brown and Jensen, 2001; Clauss, 1999, 2002; Contento et al., 2001; Johannessen and Swan, 2001; Stansberg, 2001). Water waves in all experiments are generated mechanically with different frequencies and directions. 


\section{Probabilistic Approach}

Due to strong dispersion of the water waves, each individual sine wave travels with a frequency dependent velocity, and they can travel along different directions. Due to nonlinearity of the water waves, individual sine waves interact each to other generating new spectral components. As a result, the wave field gives rise to an irregular sea surface that is constantly changing with time. To model irregular wave fields, often a random approach is used: an infinite sum of sinusoidal waves with different frequencies and with random phases and amplitudes. In the first (linear) approximation, the random wave field can be considered as a stationary random normal (Gaussian) process with the probability density distribution,

$$
f(\eta)=\frac{1}{\sqrt{2 \pi} \sigma} \exp \left(-\frac{\eta^{2}}{2 \sigma^{2}}\right)
$$

where $\eta$ is the sea level displacement with zero mean level, $\langle\eta\rangle=0$, and $\sigma^{2}$ is the variance, computed from the frequency spectrum, $S(\omega)$

$$
\sigma^{2}=<\eta^{2}>=\int_{0}^{\infty} S(\omega) d \omega
$$

It is clear, that all these formulas are valid for a stationary random process, what does not hold true in reality. Especially for freak waves due to the rarity of this event, it is hard to say if this process is stochastic or deterministic. Nevertheless, first we will discuss the freak wave formation and prediction using the Gaussian statistics. Typically, the wind wave spectrum is assumed to be narrow, thus the probability function of the wave heights will be defined through the Rayleigh distribution

$$
P(H)=\exp \left(-\frac{H^{2}}{8 \sigma^{2}}\right) .
$$

The probability that wave heights will exceed a certain level, $H$, is given by (3.3).

In oceanography, the wind wave record is characterized by the significant wave height, $H_{s}$, which is defined as the average of the higher one-third of wave heights in time series. Using the Rayleigh distribution, the significant wave height is (Massel, 1996) 


$$
H_{s}=(3 \sqrt{2 \pi} \operatorname{erfc}(\sqrt{\ln 3}+2 \sqrt{2 \ln 3}) \sigma \cong 4 \sigma
$$

where $\operatorname{erfc}(z)$ is the error function. As a result, the Rayleigh distribution can be rewritten through the significant wave height

$$
P(H)=\exp \left(-\frac{2 H^{2}}{H_{s}^{2}}\right)
$$

Mathematically, a freak wave characterized by the height, $H_{f}$, is determined from

$$
H_{f}>2 H_{s}
$$

and the amplitude criterion is used only. The probability of its formation can be evaluated from (3.5), and this dependence is presented in Figure 3.1. According to it, the probability of extreme wave formation is no more than $P\left(2 H_{s}\right)=0.000336$ or one wave among 3000 waves. Taking into account that the period of wind-generated waves is close to $10 \mathrm{~s}$, we expect a freak wave event each $8-9$ hours. According to data by Sand et al. (1990), maximum height of freak waves is $3 H_{s}$. The probability of this event is $1.5 \times 10^{-8}$, or one wave from $67,000,000$ waves. Such a wave can appear during a continuous 21-year storm.

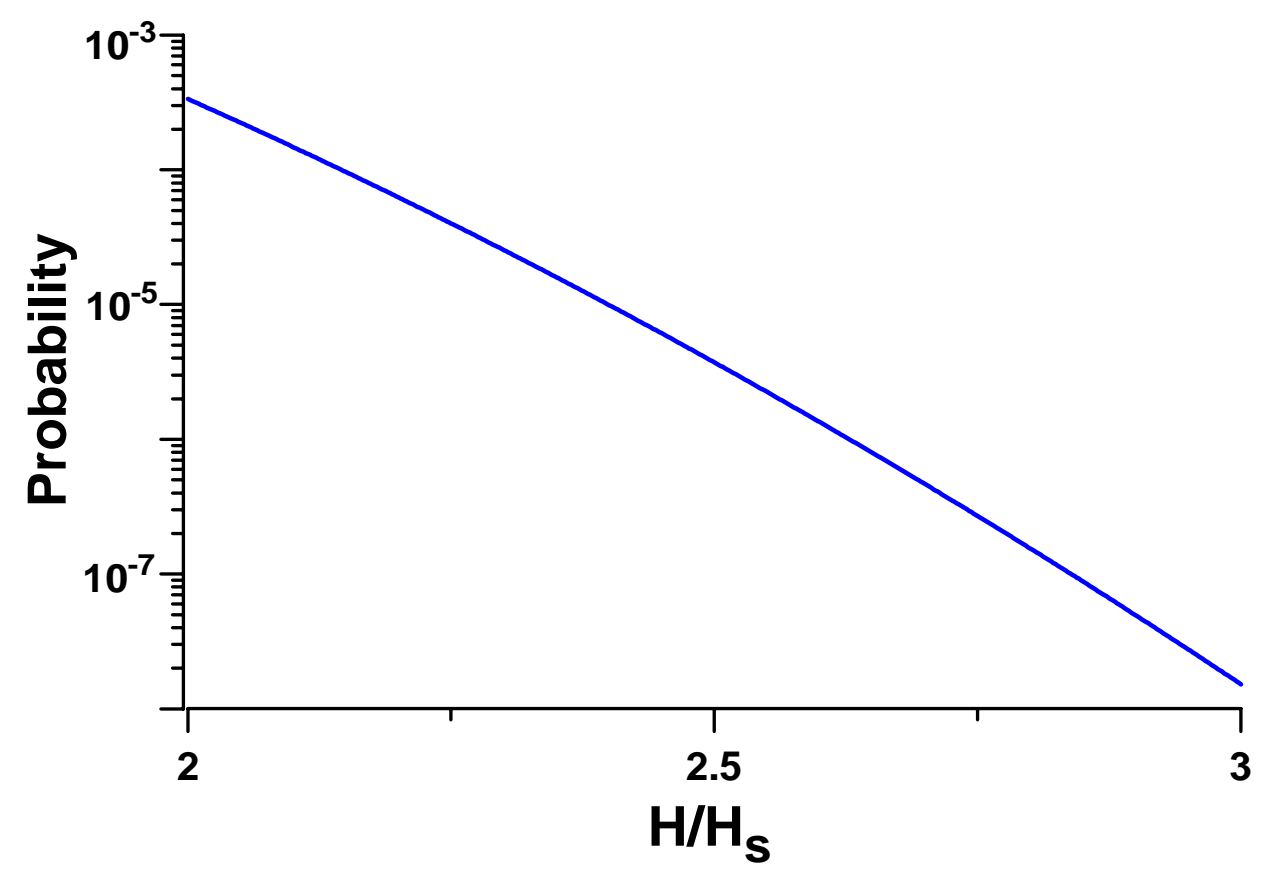

Figure 3.1. Probability of the freak wave formation 
Formula (3.6) could be simply interpreted. If we choose a wave with a maximal height $H_{\max }$ in a group of $N$ waves, its probability will be $P=1 / N$. Substituting it to (3.5), the last one could be rewritten as (Massel, 1996)

$$
H_{\max } \cong \sqrt{\frac{\ln N}{2}} H_{s}
$$

This dependence is presented in Figure 3.2. From this relation it follows that increasing the record length (number of waves) weakly influences the maximal amplitude growing. The analysis of the short time record will not give true prediction of abnormal wave formation. Thus for more reliable prediction of freak waves it is necessary to consider a large number of waves (more then 10000). In context of the water wave theories it means that numerical simulation of the freak wave phenomenon should be made on wide numerical domains with large number of realizations.

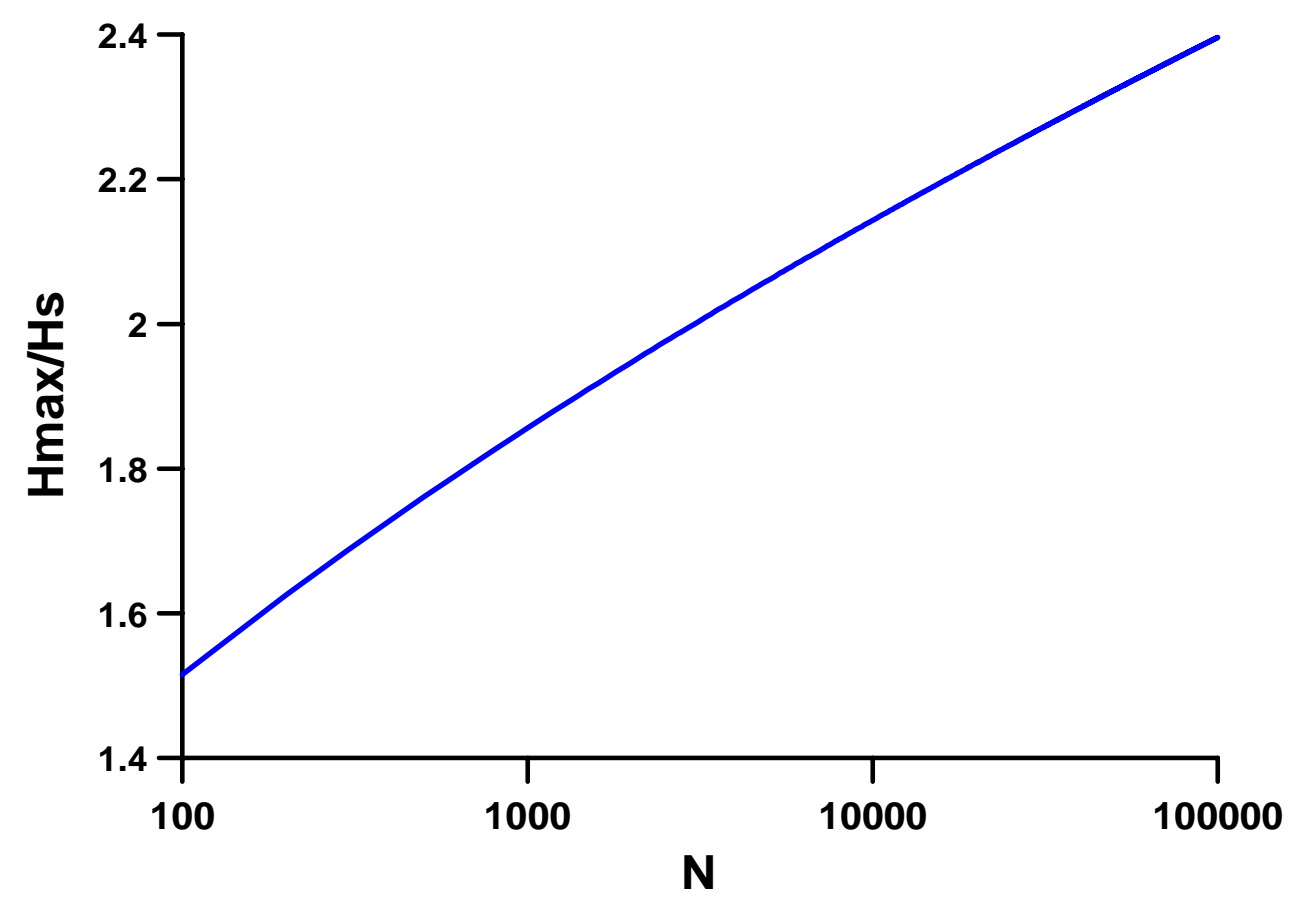

Figure 3.2. Relation between maximal wave height and number of waves in a group

It is obvious that the rare observed abnormal waves, as any distribution function tails, usually do not satisfy the statistical hypothesis the waves properties are based on. First of all, the wind wave spectrum is not very narrow as it is assumed for the Rayleigh distribution. The analysis of the distribution of the maxima (heights, crest amplitudes, trough depths) even in a Gaussian random field is a difficult mathematical task; see for instance, Boccotti (1981), Phillips et al. (1993), Boccotti (1997), Azais and Delmas (2002), and papers cited here. The second reason is the wave 
nonlinearity that leads to non-gaussian distribution functions. For instance, the measurements of freak waves in the Japan Sea (Mori et al., 2002) show a difference with the Gaussian distribution: the skewness, $\mu_{3}=0.25-0.4$, and the kurtosis, $\mu_{4}=3.1-3.4$. The third one is the atmospheric pressure and wind flow above the sea surface; they vary with time, destroying the hypothesis of the stationary random process. As a result, the Rayleigh distribution (3.5), according to the observed data, over predicts the probabilities of the highest waves (Massel, 1996; Kokorina \& Pelinovsky, 2002). Using the observation data (relatively short) it seems an impossible task to estimate the low probability of the abnormal high waves correctly. However existing models of water waves may be helpful to understand the physical mechanisms of the freak wave phenomenon and to select areas with the highest or lowest values of the rogue wave probability depending on hydrological and meterological conditions in such zones.

\section{Linear mechanisms of the rogue wave phenomenon}

In linear theory, the wind wave field can be sought as the sum of a very large number of smallamplitude independent monochromatic waves with different frequencies and directions of propagation. In statistical description, the phases of all monochromatic waves are random and distributed uniformly, providing the stationary Gaussian process in average due to the central limit theorem. The existence of rare extreme wave events (tails of the distribution function) can be interpreted as the local intercrossing of a large number of monochromatic waves with appropriate phases and directions (space-time caustics). For unidirectional wave field, the enhanced displacement can be achieved when a long wave overtake short waves due to frequency dispersion. In real three-dimensional field of water waves, both dispersion and spatial (geometrical) focusing can generate localized extreme waves. Suitable physical mechanisms are described below.

\subsection{Dispersion enhancement of transient wave groups (spatio-temporal focusing)}

If during the initial moment the short waves with small group velocities are located in front of the long waves having large group velocities, then in the phase of development, long waves will overtake short waves, and large-amplitude wave can appear at some fixed time owing to the superposition of all the waves located at the same place. Afterward, the long waves will be in front of the short waves, and the amplitude of wave train will decrease. It is obvious, that a significant focusing of the wave energy can occur only if all the quasi-monochromatic groups merge at a fixed location. Such an initial specific location of transient wave groups leading to freak wave formation may appear in the case of increasing wind due to the resonant character of 
the wave generation by wind. This scenario can explain why the freak wave phenomenon is a rare event with short "life time".

To emphasize the dispersive focusing of unidirectional water waves quantitatively, the kinematic equation for characteristic wave frequency, $\omega$, can be considered (Whitham, 1974)

$$
\frac{\partial \omega}{\partial t}+c_{g r}(\omega) \frac{\partial \omega}{\partial x}=0
$$

where the group velocity $c_{g r}(\omega)=d \omega / d k$ is calculated using the dispersion relation of water waves

$$
\omega=\sqrt{g k \tanh (k h)},
$$

$h$ is water depth and $g$ is the acceleration due to gravity. For the sake of simplicity we assume a constant water depth. Multiplying by $d c_{g r} / d \omega$ the equation (4.1) transforms into the universal form (Pelinovsky et al., 2000)

$$
\frac{\partial c_{g r}}{\partial t}+c_{g r} \frac{\partial c_{g r}}{\partial x}=0
$$

having evident physical sense: each spectral wave component propagates with its own group velocity. The solution of (4.3) corresponds the simple (Riemann) wave

$$
c_{g r}(x, t)=c_{0}(\xi)=c_{0}\left(x-c_{g r} t\right),
$$

where $c_{0}(x)$ describes initial distribution of the wave groups with different frequencies (group velocities) in space. The form of such a kinematic wave is continuously varied with distance (time), and its slope is calculated from (4.4)

$$
\frac{\partial c_{g r}}{\partial x}=\frac{d c_{0} / d \xi}{1+t d c_{0} / d \xi} .
$$

The case $d c_{0} / d \xi<0$ (or $d c_{0} / d x<0$ at $t=0$ ) corresponds to long waves behind short waves; and the initial increase of the slope of the kinematic wave up to infinity with following decrease, 
corresponds to the process of long waves overtaking short waves. The merging of several wave groups with different frequencies at the same point and time (wave focusing) appears for time, $T_{f}$ $=1 / \max \left(-d c_{0} / d x\right)$. It is obvious that several focusing points are possible for arbitrary transient wave group. The case, when all wave groups will meet at the same point, $x_{0}$, for time, $T_{f}$, is described by the self-similar solution of (4.3)

$$
c_{g r}=\frac{x-x_{0}}{t-T_{f}}
$$

Because the group velocity of the water waves varies from $(g h)^{1 / 2}$ to zero (if capillary effects are neglected), the zone of the variable wave group compresses from $(g h)^{1 / 2} T_{f}$ to zero for fixed time, $T_{f}$. The corresponding variation of the wave frequency (wave number) in the group required for optimal focusing can be easily found from (4.6). For instance, for the deep water case $\left(c_{g r} \sim 1 / \omega\right)$ it follows from (4.6) that the paddle in the laboratory tank should generate a wave train with a variable frequency, $\omega \sim\left(t_{0}-t\right)$ necessary to provide the maximum effect (optimal focusing).

The wave amplitude satisfies the energy balance equation (Whitham, 1974)

$$
\frac{\partial A^{2}}{\partial t}+\frac{\partial}{\partial x}\left(c_{g r} A^{2}\right)=0
$$

and its solution is found explicity,

$$
A(x, t)=\frac{A_{0}(\xi)}{\sqrt{1+t\left(d c_{0} / d \xi\right)}},
$$

where $A_{0}(x)$ is an initial distribution of wave amplitude in space. At each focal point, the wave becomes extreme, having infinite amplitude (near the focal point, $A \sim\left(T_{f}-t\right)^{-1 / 2}$ ).

Taking into account that each realization of wind waves always turns into frequency and amplitude modulated wave groups, and that kinematic approach predicts infinite wave height at caustics point, the probability of freak wave occurence should be very high. In fact, the situation is more complicated. Kinematic approach assumes slow variations of the amplitude and frequency (group velocity) along the wave group, and this approximation is not valid in the vicinity of the focal points (we will not discuss in this section possible limitations of wave amplitude related with nonlinear effects and wave breaking). It is a well-known problem in the ray methods, not only for water waves. Generalizations of the kinematic approach in linear 
theory can be done by using various expressions of the Fourier integral for the wave field near the caustics. In a generalized form it was expressed through the Maslov integral representation, described in details for water waves by Dobrokhotov (1983), Lavrenov (1998a), Brown (2000, 2001) and Dobrokhotov \& Zhevandrov (2003). For instance, Brown (2001) pointed out the relation between the focusing of unidirectional wave field and "canonical" caustics: fold and longitudinal cusp. We consider here the simplified form of such a representation for conditions of optimal focusing (4.6) and use the standard form of the direct and inverse Fourier transformation for water wave displacement,

$$
\begin{gathered}
\eta(x, t)=\int_{-\infty}^{+\infty} \eta(k) \exp (i(k x-\omega t) d k, \\
\eta(k)=\frac{1}{2 \pi} \int_{-\infty}^{+\infty} \eta_{0}(x) \exp (-i k x) d x,
\end{gathered}
$$

where $\eta_{0}(x)=\eta(x, 0)$ is the initial water displacement in unidirectional wave field, and $\omega$ is the wave frequency satisfying (4.2). First of all, let us re-formulate the physical problem of the freak wave formation from "normal wave field" to the mathematical problem of the appearance of singularities from smooth initial data. Due to invariance of the Fourier integral to the signs of coordinate, $x$, and time, $t$, this problem has a link with the mathematical theorem of smooth solutions of the Cauchy problem for singular initial data. For water waves the answer is positive, and the singular delta-function disturbance (as a model for freak wave) transforms into a smooth wave field (Green's function); it can be described by the asymptotic expression within the stationary-phase method

$$
\eta(x, t)=Q \sqrt{\frac{c_{g r}}{2 \pi x\left|d c_{g r} / d k\right|}} \exp (i(k x-\omega t-\pi / 4)),
$$

where the group velocity, $\mathrm{c}_{\mathrm{gr}}$ (and also wave frequency and wave number) is calculated from the conditions for optimal focusing (4.6) for fixed coordinate, $x$, and $Q$ is the intensity of the deltafunction. In the vicinity of the leading wave $(k \rightarrow 0)$ expression (4.11) is not valid (the wavelength is comparable to the distance to the source) and should be replaced with 


$$
\eta(x, t)=Q\left(\frac{2}{c t h^{2}}\right)^{1 / 3} A i\left[\left(\frac{2}{c t h^{2}}\right)^{1 / 3}(x-c t)\right]
$$

derived from (4.9) by using the long-wave approximation of the dispersion relation

$$
\omega=\sqrt{g h}\left(1-\frac{k^{2} h^{2}}{6}\right)
$$

Here $\operatorname{Ai}(z)$ is the Airy-function. As a result, the amplitude of the leading wave decreases as $t^{-1 / 3}$, and its length increases as $t^{1 / 3}$.

So, the delta-function disturbance evolves in a smooth wave field, and due to invariance with respect to coordinate and time, we may say that the initial smooth wave field like (4.11) and (4.12) with inverted coordinate and time will form the freak wave of infinite height. These solutions demonstrate obviously which wave packets can generate a freak wave in the process of wave evolution. Bona \& Saut (1993) showed that the singularity (dispersive blowup) can be formed in the long-wave approximation from the following continuous function, having the finite energy $(1 / 8<m<1 / 4)$

$$
u(x)=\frac{A i(-x)}{\left(1+x^{2}\right)^{m}}
$$

Generally speaking, the singular solutions of the linearized equations have mathematical interest only. Integral (4.9) can be calculated for smooth "freak waves" (initial data), for instance for a Gaussian pulse with amplitude, $A_{0}$ and width, $K^{-1}$, in the long-wave approximation (Pelinovsky et al, 2001b)

$$
\eta_{0}(x)=A_{0} \exp \left(-K^{2} x^{2}\right)
$$

then

$$
\eta(x, t)=\frac{A_{0}}{K \sqrt[3]{\frac{h^{2} c t}{2}}} \exp \left\{\frac{1}{2 h^{2} c t K^{2}}\left(x-c t+\frac{6}{77 h^{2} c t K^{4}}\right)\right\} \times \mathrm{Ai}\left\{\frac{x-c t+\frac{9}{77 h^{2} c t K^{4}}}{\sqrt[3]{\frac{h^{2} c t}{2}}}\right\} .
$$


Inverting coordinate and time, this wave packet evolves into a Gaussian pulse (4.15), and then again disperses according to (4.16). Figure 4.1 shows the freak wave formation in a dispersive wave packet on shallow water. Similar solutions can be found for wave packet with a gaussian envelope in deep water (Clauss \& Bergmann, 1986; Clauss, 1999; Magnusson et al., 1999)

$$
\begin{gathered}
\eta(x, t)=\frac{A_{0}}{\left(1+16 \Omega^{4} x^{2} / g\right)^{1 / 4}} \exp \left(-\frac{\Omega^{2}}{1+16 \Omega^{4} x^{2} / g}\left(t-x / c_{g r}\right)^{2}\right) \times \\
\quad \times \cos \left(\frac{\omega_{0}\left(t-x / c_{p h}\right)}{1+16 \Omega^{4} x^{2} / g}+\frac{4 \Omega^{4} x t^{2}}{g\left(1+16 \Omega^{4} x^{2} / g\right)}-\frac{1}{2} \operatorname{atan}\left[\frac{4 \Omega^{2} x}{g}\right]\right),
\end{gathered}
$$

where $A_{0}$ is the wave train amplitude, $\Omega_{0}$ and $\omega_{0}$ are frequencies of the wave envelope and carrier wave respectively. Expression (4.17) describes the evolution of a gaussian impulse from a fixed point: $x$ in (4.17) is the distance from this point. Such a situation can be modeled in the laboratory tank, see for instance, Clauss \& Bergmann, 1986; Clauss, 1999.
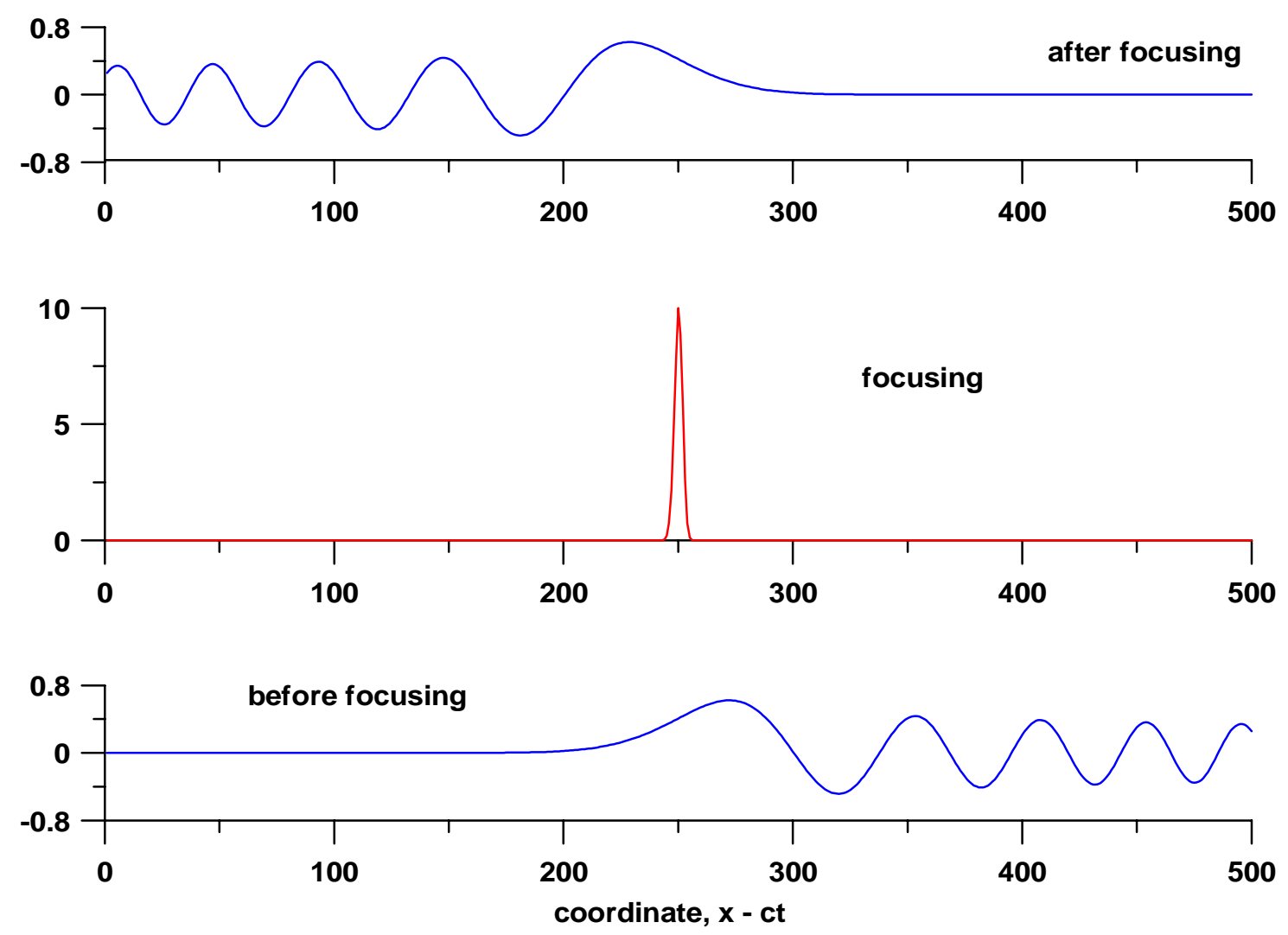

Figure 4.1. Formation of the freak wave of Gaussian form in shallow water

Exact solutions can be used for seakeeping tests or simulations of design of storm waves in ocean engineering. It is evident that in the framework of the linear theory it is easy to make a 
freak wave of any form: symmetric crest, hole in the sea, wave having a steeper forward face preceded by a deep trough (such form is used in some descriptions of the freak waves; see for instance, Lavrenov, 1998a,b).

It is important to emphasize that the dispersive focusing is the result of the phase coherence of spectral components of the wave groups, and it cannot be obtained in the framework of the models (like kinetic equations) where the wave field is the superposition of Fourier components with random phases.

\subsection{Spatial (geometrical) focusing of water waves}

Considering two horizontal coordinates, $x$ and $y$, wave frequency and wave vector should satisfy the generalized kinematic equations (4.1); see Whitham (1974)

$$
\frac{\partial \vec{k}}{\partial t}+\nabla \omega=0, \quad \nabla \times \vec{k}=0
$$

following from the definitions of the frequency and wave vector

$$
\omega=-\frac{\partial \theta}{\partial t}, \quad \vec{k}=\nabla \theta
$$

where $\theta$ is the phase of quasi-monochromatic wave: $\eta=A(x, y, t) \exp (i \theta(x, y, t))$. These equations can be rewritten in the characteristic form

$$
\frac{d \vec{r}}{d t}=\frac{\partial \omega}{\partial \vec{k}}, \quad \frac{d \vec{k}}{d t}=-\frac{\partial \omega}{\partial \vec{r}}
$$

where the wave frequency, $\omega$, satisfies the dispersion relation (4.2) with variable depth and $\bar{r}=$ $(x, y)$. There are well-known equations of the ray theory written in Hamiltonian form. The specificity of the water waves lies in the dispersion relation (4.2); see for instance, Mei (1993). If the bottom topography is stationary, the ray pattern is stationary too and determined by both the spatial variability of the bottom and the initial front locations. It is obvious that bottom topography is important mainly for long waves, in this case the ray pattern does not depend on the frequency. 
One of the trivial examples of the ray calculations is the basin of constant depth, when all rays are straight lines

$$
y-y_{0}=\tan \phi \cdot\left(x-x_{0}\right),
$$

where the initial location of the ray corresponds to coordinates, $x_{0}, y_{0}$, and its slope to angle $\phi$. Generally, the rays are not parallel lines, forming a complex pattern with many intercrossing (caustics and focuses). Another example is the parabolic bottom topography, $h(x)=h_{0}\left(x / x_{0}\right)^{2}$, when the rays are arcs of circle

$$
\left(y-y_{0}-x_{0} \tan \phi\right)^{2}+x^{2}=x_{0}^{2} / \cos ^{2}(\phi)
$$

with the center on the coastal line. For real bottom topography the ray pattern is more complicated as described by Figure 4.2, where the rays are calculated for the Japan (East) Sea from isotropic source (Choi et al., 2002). The ray theory in physics is very well developed; the classification of the caustics for water waves has been done, for instance, by Brown (2001).

Wave amplitude can be calculated from the $2 \mathrm{D}$ version of the energy balance equation

$$
\frac{\partial A^{2}}{\partial t}+\nabla \cdot\left(\vec{c}_{g r} A^{2}\right)=0
$$

which transforms into the energy flux conservation along the ray tube (Mei, 1983; Brown, 2000)

$$
c_{g r} \Lambda A^{2}=\text { const }
$$

Here $\Lambda$ is the differential width of the ray defined as the distance between neighbour rays. At any focal point, $\Lambda=0$, and, therefore, wave amplitude becomes infinite (extreme wave event).

In fact, the situation is more complicated because the energy balance equation (4.23) is no more valid in the vicinity of the caustics due to the fast variation of the wave parameters. Detailed description of the wave field in the caustics vicinity can be done by using the asymptotic Maslov representation (Peregrine \& Smith, 1979; Lavrenov, 1998a; Brown, 2000, 2001) or exact solutions for some test cases. If for instance, $h=h(x)$ only, the shallow water wave is described by the ordinary differential equation 


$$
g \frac{d}{d x}\left[h(x) \frac{d \eta}{d x}\right]+\left[\omega^{2}-g h(x) k_{y}^{2}\right] h=0
$$

where the wave is assumed to be monochromatic with frequency, $\omega$, and wave number, $k_{y}$ in $y$ direction. Caustics location can be found from (4.25) when the second bracket vanishes; let $h=$ $h_{c}$ at $x=0$. In the vicinity of caustics, the simplified expansion for depth is $h(x)=h_{c}(1+x / L)$.

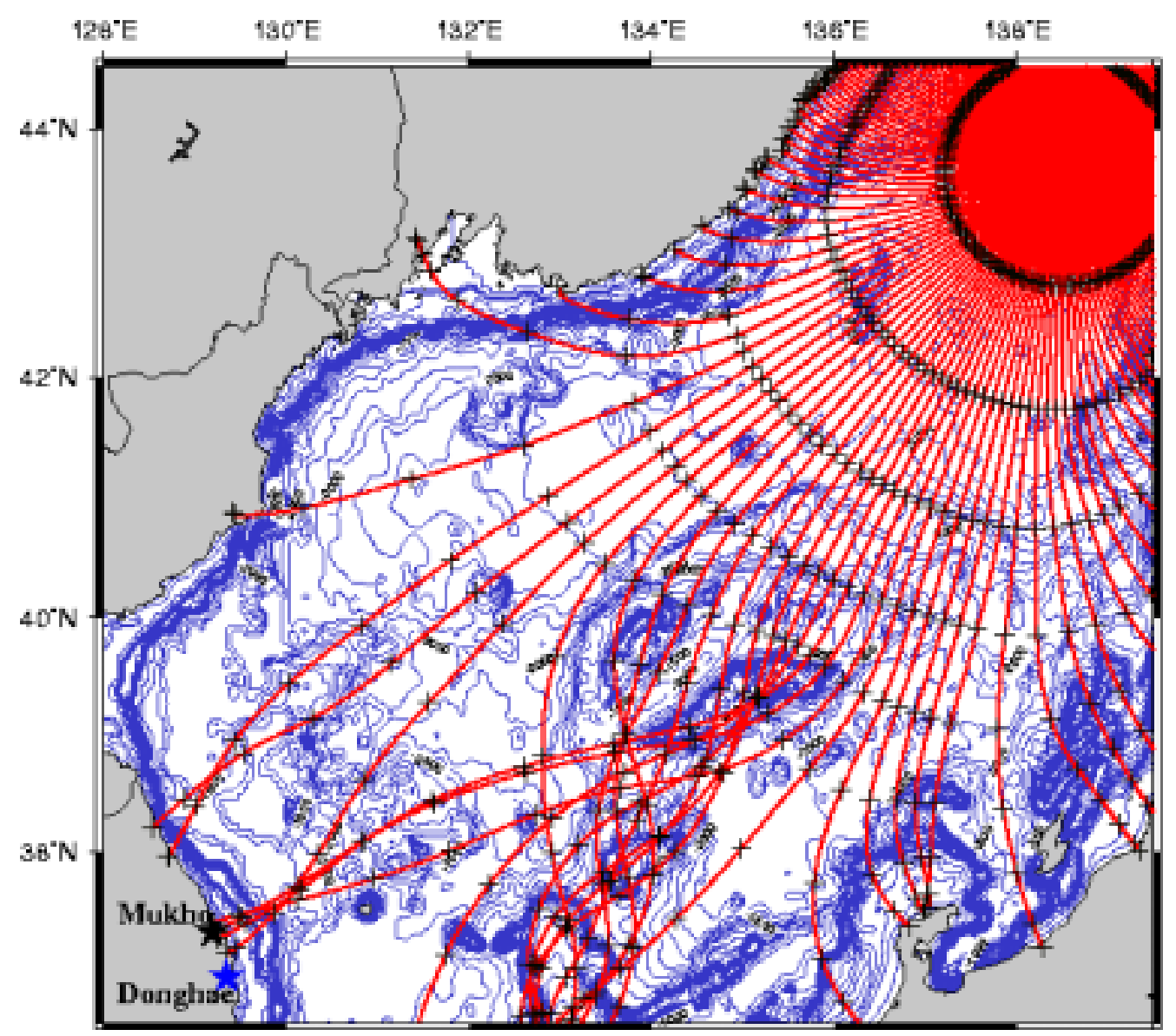

Figure 4.2. The ray pattern calculated from isotropic source in the Japan Sea

Thus, equation (4.25) in the vicinity of this point has the form of the Airy equation

$$
\frac{d^{2} \eta}{d x^{2}}-\frac{k_{y}^{2}}{L} x \eta=0
$$

and its solution is described by the Airy function

$$
\eta(x)=\text { const } \cdot A i\left(-\frac{x k_{y}^{2 / 3}}{L^{1 / 3}}\right) .
$$


As a result, the wave field is bounded on the caustics. Using asymptotic expression for the Airy function far from the caustics, the constant in (4.27) can be determined through the amplitude of the incident wave, $A_{0}$, and therefore, the wave amplification on the caustics is

$$
\frac{A_{c}}{A_{0}} \sim\left(L k_{y}\right)^{1 / 6}
$$

and it is relatively weak for long waves. It is important to conclude from the asymptotics of the test solution (4.27) that the amplitude of the wave reflected from caustics is that of the incident wave, but the phase contains the term $\pi / 4$ with the opposite sign. As a result, the phase shift between reflected and incident waves is proportional to $\pi / 2$ and this is fundamental for investigation of the solitary-like wave transformation on the caustics (additional term proportional to the travel time can be cancelled by changing time). Such a phase shift that is equivalent to Hilbert transformation, radically changes the wave shape (Pelinovsky, 1996). So, the spatial focusing produces not only a wave amplification, but also a change of the wave shape. Additionally, the wave dispersion leads to different locations of the caustics of spectral wave components and different spectral widths.

The behavior of the rays in basins with real topography is very complicated; see for example Figure 4.2. As a result, many caustics are formed in real wave fields. The general theory of the caustics is described by Arnold (1990). Very often the ray pattern can be considered as random. The statistical characteristics of the caustics in random media are investigated by Klyatskin (1993). Chaotic ray patterns may appear in deterministic medium also because the rays are described by nonlinear system of second order with variable coefficients (4.20). Such a system may have statistical behavior for specific conditions when the wave can be trapped (Abdullaev, 1991; Pelinovsky, 1996).

Formally, the caustics of monochromatic wave fields in basins with stationary bottom topography exist for infinite long time. In fact, a variable wind generates complex and variable structures of rays in storm areas, playing the role of initial conditions for the system (4.20). It is evident that caustics are very sensitive to the small variation of the initial conditions, and as a result, the caustics and focuses appear and disappear at "random" points and "random" times, providing rare and short-lived character of the freak wave phenomenon.

We would like to point out also that waves can be trapped in coastal zones. Such waves are dispersive even in the long-wave approximation, and they may give a spatial-temporal focus (Kurkin \& Pelinovsky, 2002). 


\subsection{Wave-current interaction as a mechanism of freak waves}

Noting that rogue waves were observed very often in such strong currents as Gulf Stream and Agulhas Current, the problem of the wave-current interaction requires a special investigation (Peregrine, 1976; Lavrenov, 1998a,b; White \& Fornberg, 1998; Brown, 2000, 2001). Formally, the ray pattern is described again by the system (4.20) where the dispersion relation should be corrected. Considering the deep water waves case, the dispersion relation for waves on a steady current becomes anisotropic, see Figure 4.3 for unidirectional wave propagation

$$
\omega=\Omega(k)+\vec{k} \vec{U}(x, y), \quad \Omega= \pm \sqrt{g k} .
$$

Even in one-dimensional case, with $U_{x}(x)$ only, the wave-current interaction is not trivial. When the current is opposite to incident monochromatic wave, it blocks the wave at the point, $x_{0}$, where the group velocity (in non-moving system of coordinates) is zero,

$$
c_{g r}=\frac{d \omega}{d k}=\frac{1}{2} \sqrt{\frac{g}{k}}+U\left(x_{0}\right)=0 .
$$

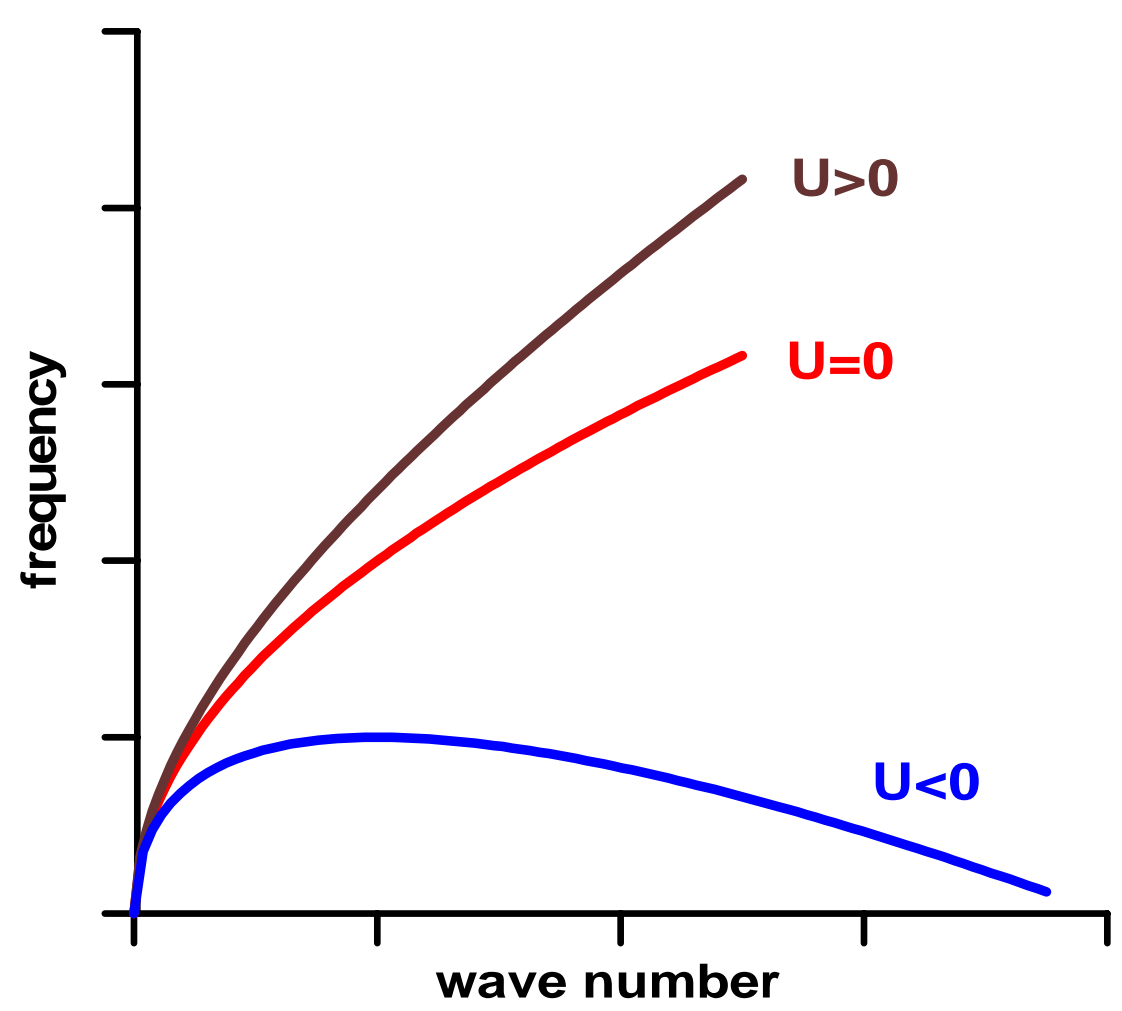

Figure 4.3. Dispersion relation for unidirectional wave propagation

Wave approaching the blocking point has the phase and group velocities of the same sign, after reflection from the blocking point the group velocity has a sign opposite to that of the phase 
velocity; see Figure 4.3. The wave number increases in the process of interaction, and an initial long wave transforms to a short wave. The wave amplitude can be found from the wave action balance equation

$$
\frac{\partial}{\partial t}\left(\frac{A^{2}}{\Omega}\right)+\nabla \cdot\left(\frac{c_{g r} A^{2}}{\Omega}\right)=0
$$

generalizing the energy balance equation (4.23) for waves on current. For steady currents, (4.31) transforms into the wave action flux

$$
c_{g r} \Lambda A^{2} / \Omega=\text { const } .
$$

where $\Lambda$ as previously is the differential width of the ray tube. For the case of unidirectional wave propagation, the blocking point, characterized by zero group velocity (4.30) plays the role of caustics and here the wave amplitude formally tends to infinity. In fact, equation (4.31) is not valid in the vicinity of the caustics, and more accurate asymptotic analysis using the Maslov representation is needed to give the following expression for the wave field (Peregrine and Smith, 1979; Lavrenov, 1998a) generalizing (4.27)

$$
\eta(x)=\text { const } \cdot A i\left[\left(\frac{8 \partial U / \partial x}{\Omega\left(k_{*}\right)}\right)^{1 / 3} k_{*}\left(x-x_{0}\right)\right] \exp \left(i k_{*} x-\omega t\right)
$$

where $k *$ is the value of the wave number at the blocking point determined by (4.30) and $\partial U / \partial x$ is calculated at the same point. As a result, wave amplitude at the blocking point is bounded; compare with (4.28)

$$
\frac{A_{c}}{A_{0}} \sim\left(\frac{\Omega}{d U / d x}\right)^{1 / 6}
$$

Reflection of oblique wave by currents was recently studied analytically by Shyu \& Tung (1999). A more general approach takes into account two-horizontal coordinates and real profiles of transverse shear currents for the complex ray pattern with generation of "normal" caustics when the differential width is zero $(\Lambda=0)$, and specific "current" caustics when $c_{g r}=0$. Lavrenov (1998a,b) calculated the ray pattern in the vicinity of the Agulhas Current for one 
event of freak wave occurence and showed that it contains focus points where the wave energy concentrates. White and Fornberg (1998) took into account the weak randomness of the current and showed that the distribution of the focus points tends to the universal curve. These calculations demonstrate that variable currents can lead to the formation of rogue waves and the authors of the above cited papers assume that wave-current interaction is the major mechanism of the rogue wave phenomenon in deep water. The short-lived character of the freak waves on current can be provided by time variation of the current and wind.

It is important to mention that caustics in the wave field on the current are mainly dispersive, and this should influence significantly the solitary-like pulse propagation.

\subsection{Atmospheric forcing}

Caustics described above appear in the process of the free wave evolution. An interaction of water waves with atmosphere, as it is known, can be described mainly by two mechanisms: through fluctuations of the atmospheric pressure (Phillips mechanism) and through interaction with unstable fluctuations of the shear wind flow (Miles mechanism). In general, both mechanisms can be parameterized in the energy balance equation by the terms, like $q_{p h}+q_{m i} A^{2}$, where $q_{p h}$ and $q_{m i}$ are prescribed in the framework of the linear theory of the wind wave generation. Atmospheric forcing increases the wave energy and its variability in space and time. Characteristic scales here are large enough (many wavelengths) due to weak interaction between wind flow and waves. Therefore, atmospheric forcing cannot change radically the ratio of the wave energy inside/outside the focus points. More importantly is that atmospheric forcing in storm areas determines the initial location of the wind wave directions variable with time. The ray pattern in space is very sensitive to the weak variation of initial locations of the wave rays, providing "unpredictability" in appearance and disappearance of the focus points. Mallory (1974) pointed out that according to observations, the rogue waves in the Agulhas Current frequently occur when the increased wind of north-east direction is appeared several hours before the event, and the wind changes its direction from north-east to south-east for 4 hours (see also, Lavrenov, 1998). The first factor (increasing of the wind flow) plays an important role in the mechanism of the dispersive focusing. The second one provides also the variation of the spatial focusing. Lavrenov (1998a,b) calculated the ray pattern for wind and wave conditions during one freak wave event and found the distribution of the focus points in the Agulhas Current due to the wave-guide formation. This distribution corresponds roughly to the typical locations of the observed freak waves. 
It is important also to mention that the wind flow generates generally random wave field. Due to the random orientation of the wave directions and frequency dispersion, the random caustics have to appear and disappear. But their intensity (wave energy) cannot be high, because a random number (not too large) of wave groups meet in caustics. As a result, most of the caustics in random wave fields cannot be identified with freak waves. Only if optimal conditions are fullfilled (strong temporal and spatial coherence in the wave field), the wave amplitude on the caustics exceeds twice the significant wave height (amplitude criterion for freak wave event). It explains why the probability of the rogue wave appearance is lower than the probability of the focus point appearance. Random and coherent wave components do not interact in the framework of the linear theory; therefore, the weak coherent "optimal" component can transform into the freak wave on the background of strong random field.

\section{Nonlinear theories of rogue wave occurrence}

From linear theories one may conclude that the main mechanisms of rogue wave phenomenon are related with wave focusing of frequency modulated wave groups (dispersive and geometrical focusing), and with blocking effect of spectral components on opposite currents. Both mechanisms are very sensitive to the spectrum width of the wind wave field. In particular, the focusing mechanism requires a wide energetic spectrum with a specific phase distribution; meanwhile the wave-current mechanism is effective when the spectrum is very narrow. Nonlinearity may destroy the phase coherence between spectral components, "washing out" caustics and focuses that decreases the amplitude of extreme waves (nonlinear effects on waves near caustics have ben studied by Peregrine and Smith, 1979; Peregrine, 1983a). The second important ingredient is the role of randomness of the wind wave field that also acts on the phase coherence of "deterministic transient" waves (in linear theory, deterministic and random components propagate independently). And third, nonlinearity may produces instability of the wave field leading to formation of anomalous high waves. All these aspects will be analyzed here mainly in the weakly nonlinear limit.

\subsection{Weakly nonlinear "rogue" wave packets in deep and intermediate}

\section{depths}

Simplified nonlinear model of 2D quasi-periodic deep-water wave trains in the lowest order in wave steepness and spectral width is based on the nonlinear Schrödinger equation 


$$
i\left(\frac{\partial A}{\partial t}+c_{g r} \frac{\partial A}{\partial x}\right)=\frac{\omega_{0}}{8 k_{0}^{2}} \frac{\partial^{2} A}{\partial x^{2}}+\frac{\omega_{0} k_{0}^{2}}{2}|A|^{2} A,
$$

where the surface elevation, $\eta(x, t)$ is given by

$$
\eta(x, t)=\frac{1}{2}\left(A(x, t) e^{i\left(k_{0} x-\omega_{0} t\right)}+c . c .+\ldots\right),
$$

$k_{0}$ and $\omega_{0}$ are the wave number and frequency of the carrier wave, c.c. denotes the complex conjugate, and (...) determine the weak highest harmonics of the carrier wave. The complex wave amplitude, $A$, is a slowly varying function of $x$ and $t$.

The nonlinear Schrödinger equation that was derived about 40 years ago plays an important role in the understanding of nonlinear dynamics of water waves. It is well-known that a uniform train of amplitude $A_{0}$ is unstable to the Benjamin- Feir instability (BF instability or modulational instability) corresponding to long disturbances of wave number, $\Delta k$, of the wave envelope satisfying the following relation

$$
\frac{\Delta k}{k_{0}}<2 \sqrt{2} k_{0} A_{0}
$$

The maximum instability occurs at $\Delta k / k_{0}=2 k_{0} A_{0}$, with the maximum growth rate equal to $\omega_{0}\left(k_{0} A_{0}\right)^{2} / 2$. The nonlinear stage of the BF instability was deeply investigated analytically, numerically and experimentally. Figure 5.1 illustrates the formation of high-energetic wave group in slowly modulated wave train due to the BF instability simulated numerically (Pelinovsky et al., 2001a). Wave groups appear and disappear for characteristic timescale of order $1 /\left[\omega_{0}\left(k_{0} A_{0}\right)^{2}\right]$. Such a behavior can be due to the excitation of breather solutions of the nonlinear Schrödinger equations (Peregrine, 1983b; Henderson et al., 1999; Dysthe \& Trulsen, 1999; Osborne et al., 2000). One of the breather solutions (a singular breather on an infinite domain) corresponds to the so-called algebraic breather (in the system of coordinates moving with the group velocity)

$$
A(x, t)=A_{0} \exp \left(i \omega_{0} t\right)\left[1-\frac{4\left(1+2 i \omega_{0} t\right)}{1+16 k_{0}^{2} x^{2}+4 \omega_{0}^{2} t^{2}}\right] .
$$


This algebraic breather is shown in Figure 5.2. The maximal height of this wave (from trough to crest) exceeds 3. Also breather solutions can be periodic in time (Ma-breather) and in space (Akhmediev breather); see for instance, Dysthe \& Trulsen (1999) and Osborne et al. (2000). All such solutions can be considered as simple analytical models of freak waves (in fact, it is a group of huge waves in the framework of the nonlinear Schrödinger equation) because they satisfy the amplitude criterion (3.6) for the height of rogue waves. Breather solutions describe simplified dynamics of modulationally unstable wave packets. Osborne et al., (2001) and Calini \& Schober (2002) gave more detailed analysis of rogue waves event during the nonlinear stage of modulational instability by using the inverse scattering approach (so-called homoclinic orbits).

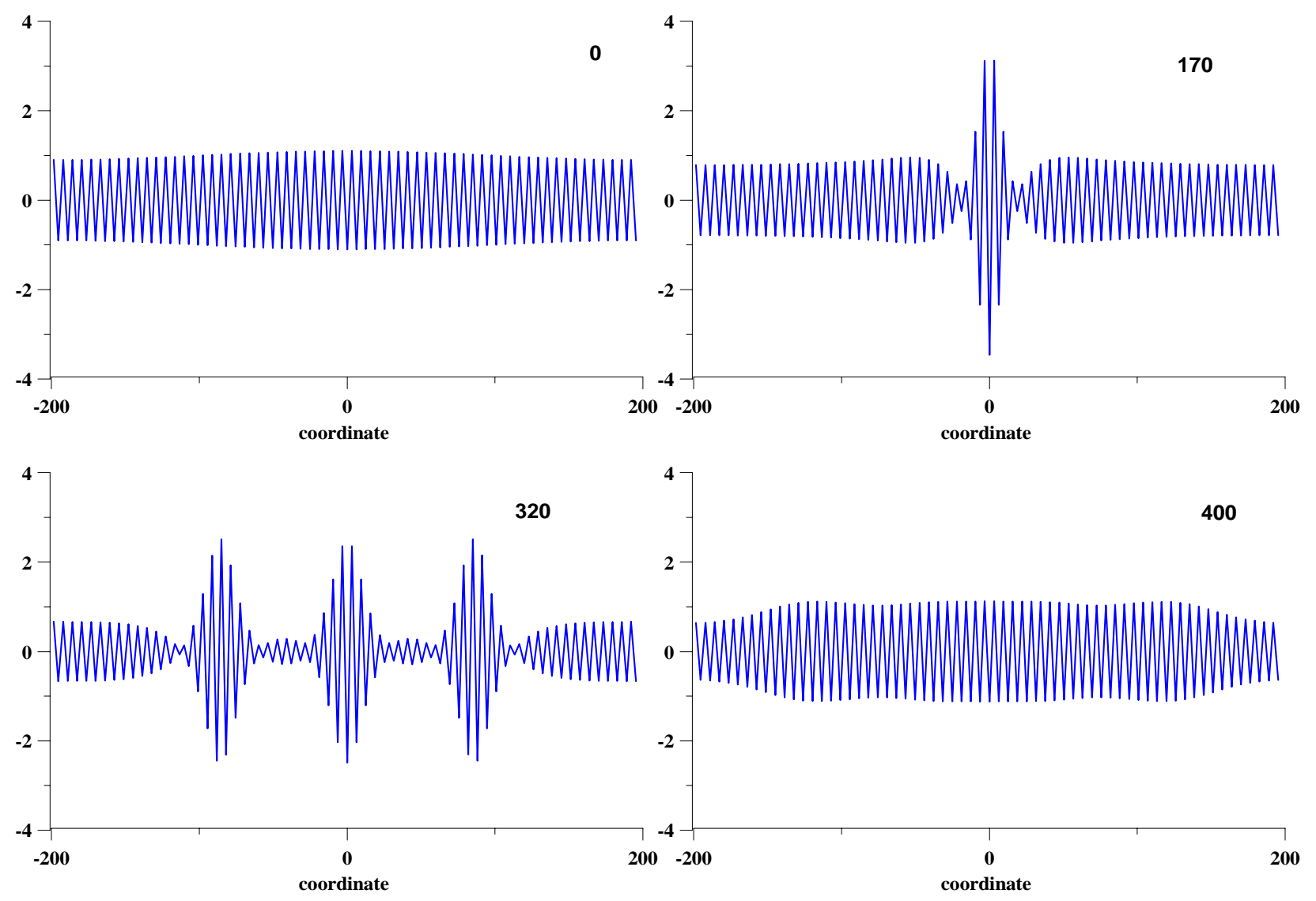

Figure 5.1. Snapshot of the evolution of weakly modulated wave train (numbers - time normalized by the fundamental wave period)

So, the nonlinear instability of a weakly modulated wave train in deep water may generate shortlived anomalous high waves, and this is a new mechanism at the origin of the rogue wave phenomenon different in principle of all mechanisms presented in section 4. 


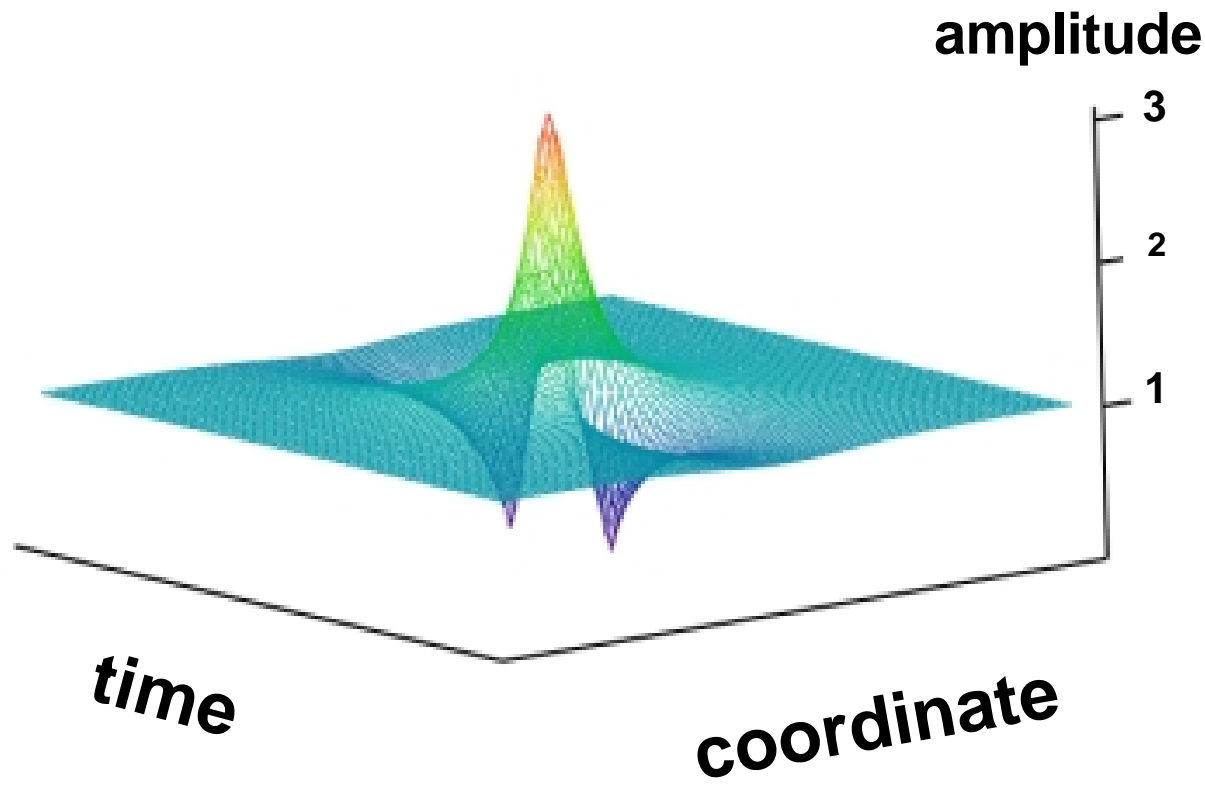

Figure 5.2. Algebraic breather as a model of abnormal wave in a time periodic wave train

If the modulation of the periodic wave train is not weak, the wave spectrum may present many harmonics contained in a relatively narrow band for applicability of the nonlinear Schrödinger equation. In this case, the wave is assumed to be the superposition of different spectral components propagating with different velocities depending on the wave number and the wave amplitude as well. As a result, the focusing process is possible for specific phase relations between harmonics. Formally, this process can be analyzed by using the generalized kinematic equations (4.1) and (4.7) with the dispersion relation of water waves depending on the wave amplitude, but this system is elliptic (Lighthill, 1965) and does not provide simple interpretation in terms of caustics as hyperbolic systems. Due to invariance of the nonlinear Schrödinger equation with respect to the sign of the coordinate and time (changing $A$ with its complex conjugate $A^{*}$ ) we may again consider the Cauchy problem for (5.1) with singular initial data, like the delta-function. Using the inverse scattering method, it can be shown (Satsuma, 1974; Kharif et al., 2001) that the delta function evolves in a smooth solution corresponding to a dispersive train and a set of solitons (if the intensity of delta function is large enough). It means that inverted smooth wave field will generate the delta function in the process of its evolution and then again will disperse. These simple arguments show that wave field may focus in the nonlinear case also, but specific conditions between phase (and amplitudes) of the dispersive trains and solitons should be provided. Figure 5.3 describes numerical simulations of the focusing of an initial wave train with weak amplitude modulation (as in Figure 5.1) and phase modulation - chirp $\left(\exp \left(i \beta x^{2}\right)\right)$, which is optimal for linear focusing (Kharif et al., 2001; 
Pelinovsky et al., 2001a). Some analytical solutions of the nonlinear Schrödinger equation for initial wave packets with chirp were obtained by Calini \& Schober (2002).

For random disturbances the situation is more complicated. First of all, as Alber (1978) pointed out randomness increases the stability of the wave packet, reducing the modulation instability. If the wave process can be represented by nearly Gaussian random functions with characteristics spectrum width, $\Delta k$ and characteristic amplitude, $A_{0}$ defined as $2\left(\left\langle\eta^{2}\right\rangle\right)^{1 / 2}$, the wave field is stable when

$$
\frac{\Delta k}{k_{0}}>2 k_{0} A_{0}
$$
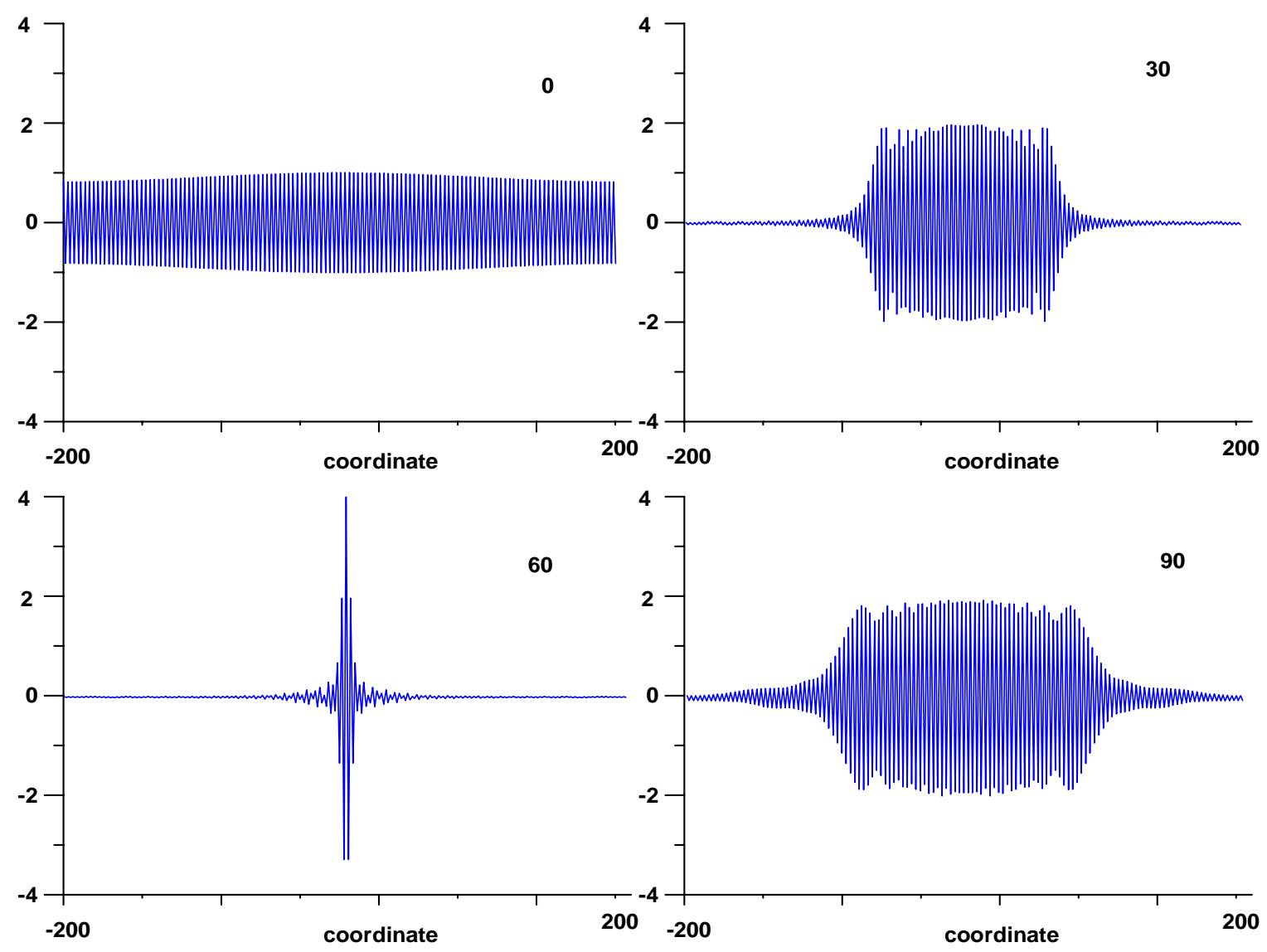

Figure 5.3. Snapshot of the evolution of wave packet with chirp train (numbers - time normalized by the fundamental wave period)

It is almost the same as for deterministic side-band disturbances; see (5.3). Dysthe et al. (2003) performed the numerical simulation of the nonlinear Schrödinger equation (5.1) with random initial profiles of Gaussian shape. The spectrum broadens symmetrically with time until it reaches a quasi-steady width. If the initial parameters of the wave field satisfy (5.5) and correspond to the stable case, they do not change in the process of the averaged wave field 
evolution. If the wave field is initially unstable, its spectrum becomes wide, reducing the instability. The final parameters of the averaged wave field again satisfy (5.5). So in average, the modulational instability is a factor of relaxation in a random wave field transforming its spectrum so that the condition (5.5) is satisfied.

In situ, wind wave realizations being uniform in average must contain both almost uniform wave trains and frequency modulated wave packets. Therefore, freak wave events can appear as the result of modulational instability and focusing. Using the JONSWAP spectrum Onorato et al (2001) performed numerical experiments to investigate freak wave generation and its statistics. In particular, it was shown that if the spectrum is narrow (increasing value of the "enhancement" coefficient in the JONSWAP spectrum) the probability of the rogue wave occurence is increased. This increase can be explained by the effect of the modulational instability in addition to the wave focusing.

The nonlinear Schrodinger equation can be derived for basins of arbitrary depth. For finite depth, the coefficients of (5.1) are function of $k h$, where $h$ is water depth. For 2D water wave fields, modulational instability occurs only for $k h>1.363$. On shallow water uniform wave trains are stable and only the focusing mechanism can be suggested for explanation of the rogue wave phenomenon. Due to weak dispersion on shallow water, the coherence between spectral components becomes strong, leading to the formation of solitons and quasi-shock waves. This requires an another model than the nonlinear Schrödinger equation and will be described in next section.

For 3D wave trains the 2D nonlinear Schrödinger equation is

$$
i\left(\frac{\partial A}{\partial t}+c_{g r} \frac{\partial A}{\partial x}\right)=\frac{\omega_{0}}{8 k_{0}^{2}} \frac{\partial^{2} A}{\partial x^{2}}-\frac{\omega_{0}}{4 k_{0}^{2}} \frac{\partial^{2} A}{\partial y^{2}}+\frac{\omega_{0} k_{0}^{2}}{2}|A|^{2} A
$$

It is important to note that the 2D nonlinear Schrödinger equation is principally anisotropic, and modulations of wave packets in the longitudinal and transversal directions behave differently, in particular modulations in the transverse direction are stable. The domain of the BF instability (modulation) of the Stokes wave of amplitude $A_{0}$ can be found very easily; see for instance, Dias and Kharif (1999), it (dashed area) is shown in Figure 5.4 (normalized by $(2)^{3 / 2} k^{2} A_{0}$ )

$$
\frac{\sqrt{2} \Delta k_{y}}{k_{0}}<\frac{\Delta k_{x}}{k_{0}}<\sqrt{\frac{2\left(\Delta k_{y}\right)^{2}}{k_{0}^{2}}+8 k_{0}^{2} A_{0}^{2}} .
$$


The most important observation is that the instability region is unbounded in the perturbation wave vector plane.

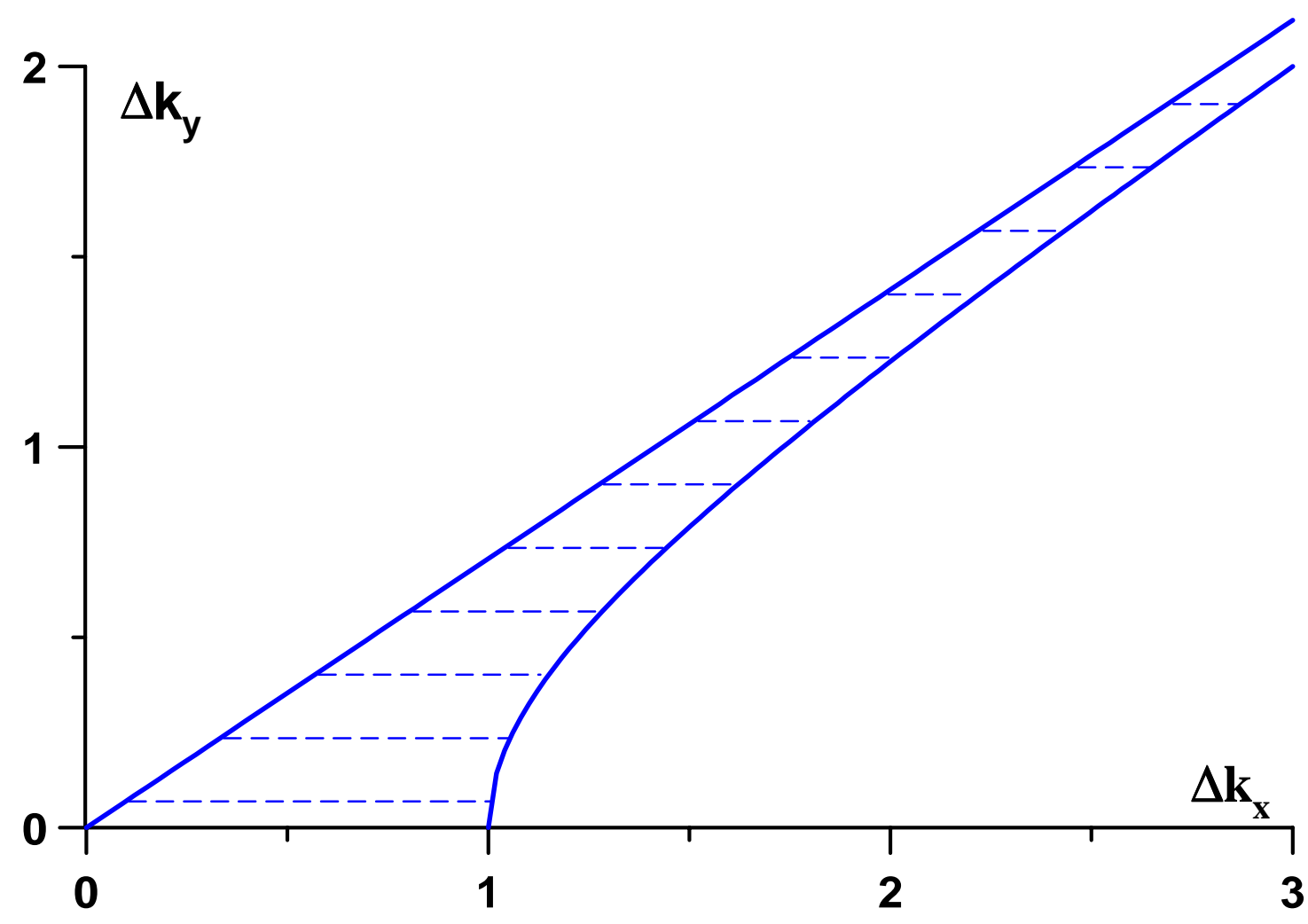

Figure 5.4. Diagram of instability of the 3D Benjamin-Feir instability in the plane of wave numbers

Therefore, one can expect that both mechanisms of rogue wave generation, modulational instability and wave focusing, should work for 3D wave trains in the deep water. The wave focusing in horizontal plane may be observed as dispersive focusing (due to wave dispersion), as well as geometrical focusing (due to different directions of wave propagation). Thus, the mechanism of rogue wave generation is richer for 3D water waves.

Figure 5.5 describes the development of the modulational instability for weak amplitude modulation $(10 \%)$ of the periodic wave train $\left(\Delta k_{x} / k=\Delta k_{y} / k=0.3\right)$; for more details see Slunyaev et al., 2002. In the first stage a quasi one-dimensional wave crest grows $(\omega t \approx 2.5)$. Then the transverse modulation becomes important $(\omega t \approx 3)$ leading first to the formation of several isolated peaks $(\omega t \approx 3.8$ ) followed by the merging of four peaks whose coupling gives rise to the giant wave $(\omega t \approx 4.1)$, depicted in Figure 5.6. Its amplitude exceeds 7 times that of the initial wave! In numerical experiments by Onorato et al. (2000) the rogue wave height reached the value $42 \mathrm{~m}$ (the maximum crest of $29 \mathrm{~m}$ is accompanied by a minimum $13 \mathrm{~m}$ deep), the wavelength and steepness are about $450 \mathrm{~m}$ and 0.33 respectively. 
The phenomenon of large-amplitude wave formation is very sensitive to random components of the wave field. The maximum wave height is reduced by $15 \%$, if the noise component has an amplitude equal to $1 \%$ of the deterministic perturbation and a spectral width 5 times wider (Slunyaev et al., 2002). It corresponds to the theoretical result by Alber (1978) that a random field tends to restabilize an almost uniform wave train. Nevertheless, our experiments showed that the randomness does not destroy the BF instability growth at all. Recently, Dysthe et al. (2003) obtained the same result from direct numerical simulations of the evolution of a narrowbanded spectrum of random waves within the framework of the $2 \mathrm{D}$ nonlinear Schrödinger equation.

To compare with modulational instability, a similar simulation was performed for dispersive focusing showing how robust to random wave field intensity is the latter mechanism. Figure 5.7 describes a rogue wave event from a random wave filed whose characteristic amplitude is 5 times greater than the amplitude of deterministic frequency modulated wave train (Slunyaev et al., 2002).
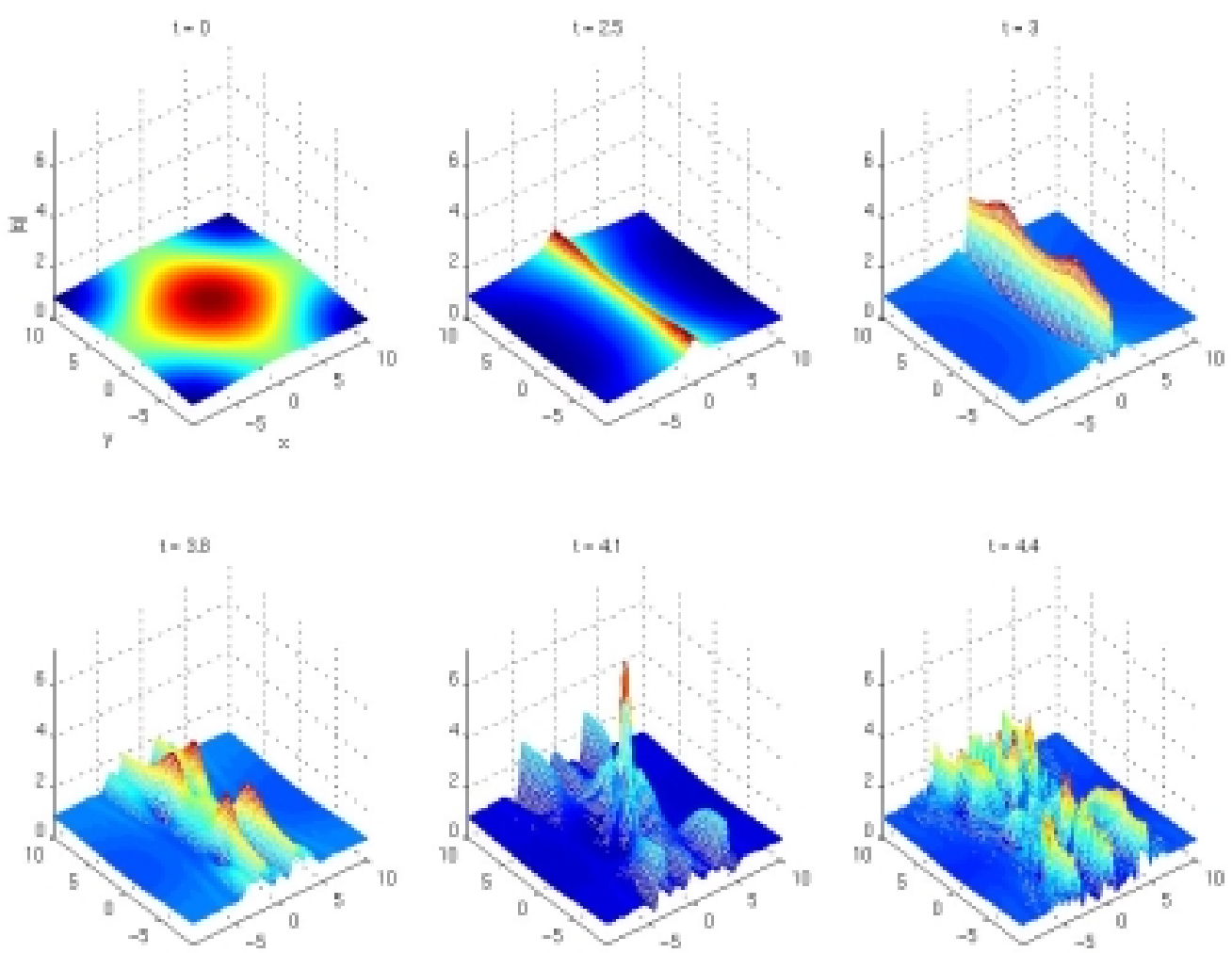

Figure 5.5. Evolution of the 3D Benjamin-Feir instability for weakly modulated wave train 


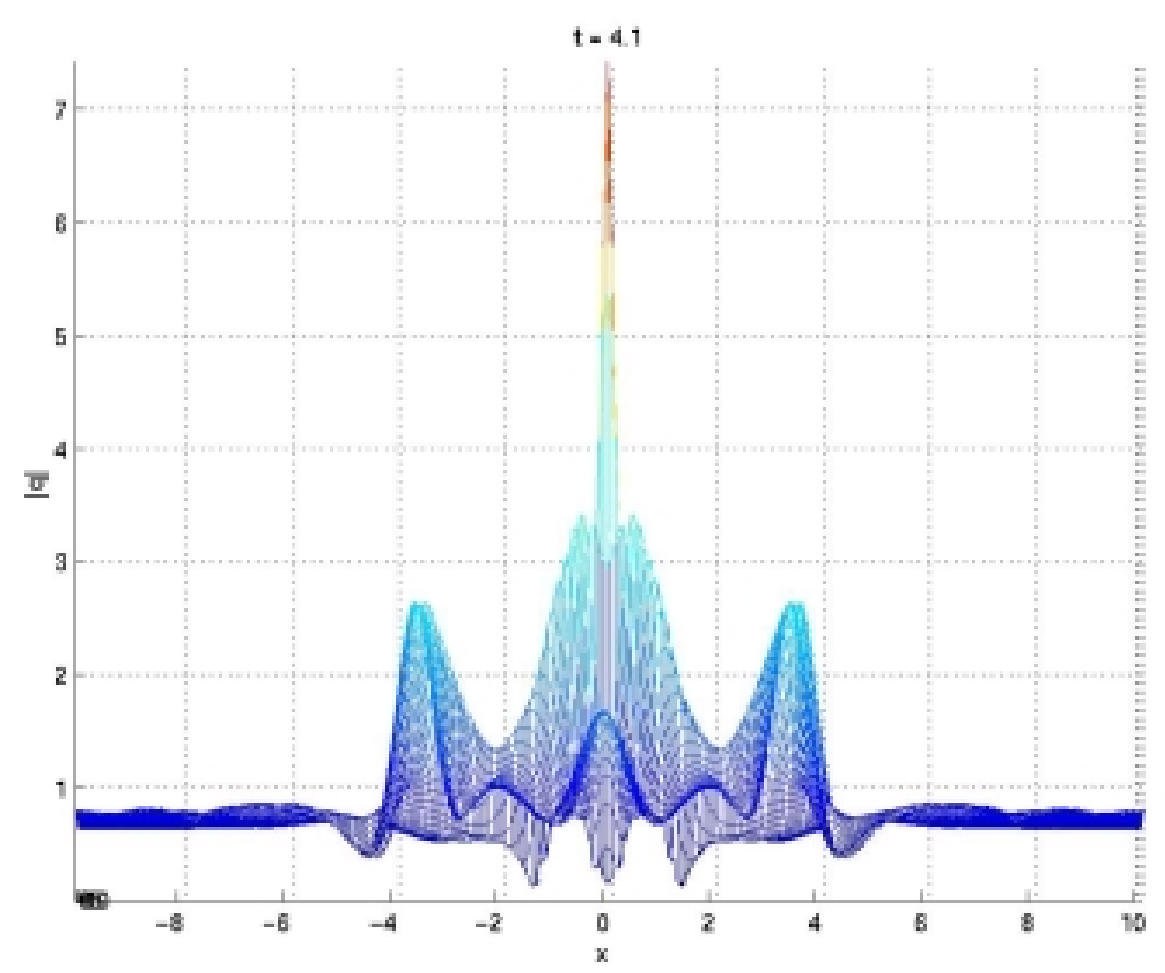

Figure 5.6. Giant wave due to 3D Benjamin - Feir instability
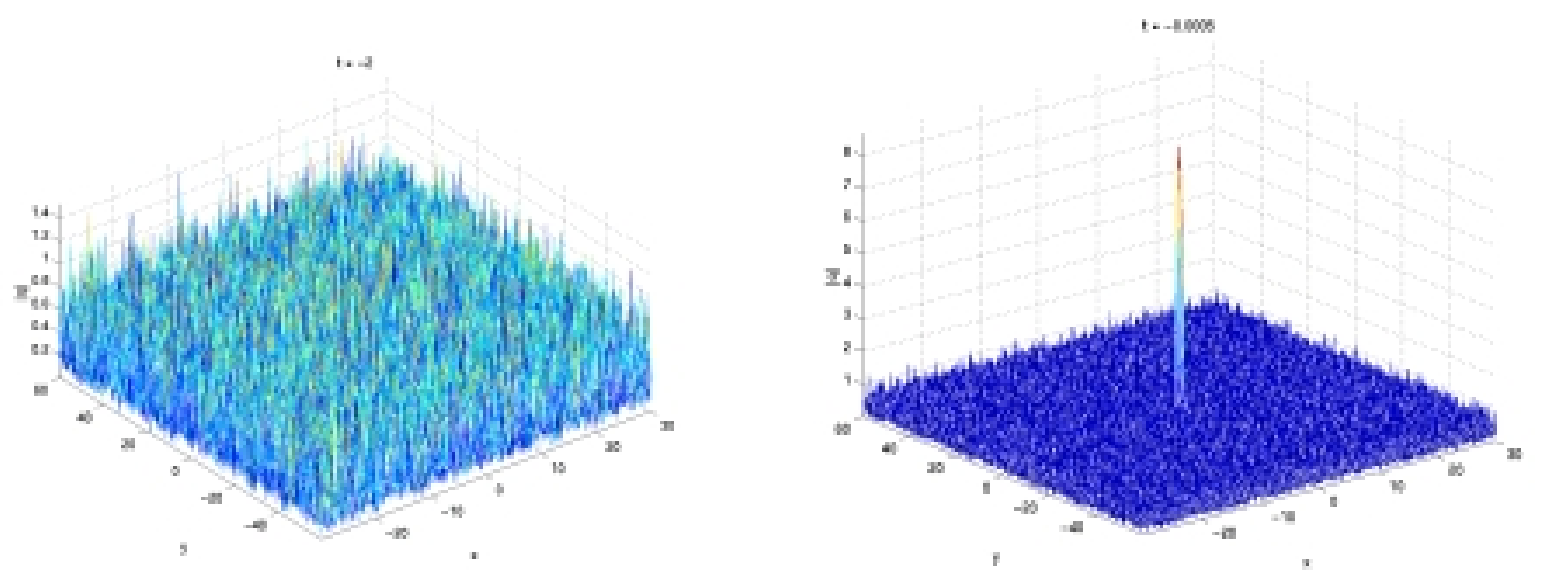

Figure 5.7. Formation of a huge wave from 3D random field

For finite depths the effect the mean flow generated by modulated waves becomes important, and the weakly nonlinear and weakly modulated 3D wave trains in water of finite depth may be described by the Davey-Stewartson system in dimensionless form

$$
\left\{\begin{array}{c}
i \frac{\partial q}{\partial t}+\frac{\partial^{2} q}{\partial x^{2}}-\frac{\partial^{2} q}{\partial y^{2}}+2 q\left(|q|^{2}-p\right)=0, \\
s_{1} \frac{\partial^{2} p}{\partial x^{2}}+s_{2} \frac{\partial^{2} p}{\partial y^{2}}=\frac{\partial^{2}}{\partial x^{2}}|q|^{2},
\end{array}\right.
$$


where

$$
\begin{gathered}
S_{1}=S_{1} \frac{N_{1}}{N_{2}^{2}}, \quad S_{2}=S_{2} \frac{N_{1}}{N_{2}^{2}} \frac{D_{1}}{D_{2}}, \quad D_{1}=-\frac{4 k}{c_{p h}} \frac{\partial c_{g r}}{\partial k}, \quad D_{2}=\frac{4 c_{g r}}{c_{p h}}, \\
N_{1}=\frac{1}{8}\left(1+9 \sigma^{-2}-13\left(1-\sigma^{2}\right)-2 \sigma^{4}\right), \quad N_{2}=1+\frac{c_{g r}}{2 c_{p h}}\left(1-\sigma^{2}\right), \\
S_{1}=\frac{k h}{\sigma}-\frac{c_{g r}^{2}}{c_{p h}^{2}}, \quad S_{2}=\frac{k h}{\sigma}, \quad \sigma=\tanh k h .
\end{gathered}
$$

$C_{g r}$ and $c_{p h}$ are group and phase velocities, and the surface elevation is given by

$$
\begin{gathered}
\eta(X, Y, T)=\frac{c_{p h}^{2}}{g \sqrt{2 N_{1}}}[q(x, y, t) \exp (i(\omega T-k X))+c . c .]+\ldots, \\
x=\frac{2 k}{\sqrt{D_{1}}}\left(X-c_{g r} T\right), \quad y=\frac{2 k}{\sqrt{D_{2}}} Y, \quad t=\frac{1}{2} \omega T .
\end{gathered}
$$

The mean flow induced by wave train is described by a function, $p$. The coefficients $N_{l}$ and $N_{2}$ are nonlinear coefficients, $D_{1}$ and $D_{2}$ are the coefficients of longitudinal and transverse dispersion. All the coefficients of the system (5.8) depend on the parameter $k h$ only. For 2D waves the Davey-Stewartson system reduces to the nonlinear Schrodinger equation (5.1), focused for deep water and defocused for shallow water. For 3D waves in deep water the mean flow is negligible, and the Davey-Stewartson system reduces to the $2 \mathrm{D}$ nonlinear Schrodinger equation (5.6). For shallow water, the Davey-Stewartson reduces to the integrable system (Anker \& Freeman, 1978).

The main feature of 3D behaviour of water waves is the existence of the BF instability for any water depth. Figure 5.8 displays the areas of instability (white) in wave number plane of the envelope for various values of $k h$. In general, unstable disturbances propagate obliquely to the direction of the carrier wave. When the depth decreases, the instability area becomes narrow and its increment decreases. Therefore, both mechanisms of rogue wave phenomenon (modulational instability and wave focusing) should work for 3D wave train in shallow water and intermediate depths. A detailed analysis of rogue wave scenarios in the framework of the Davey-Stewartson system can be found in Slunyaev et al. (2002). 

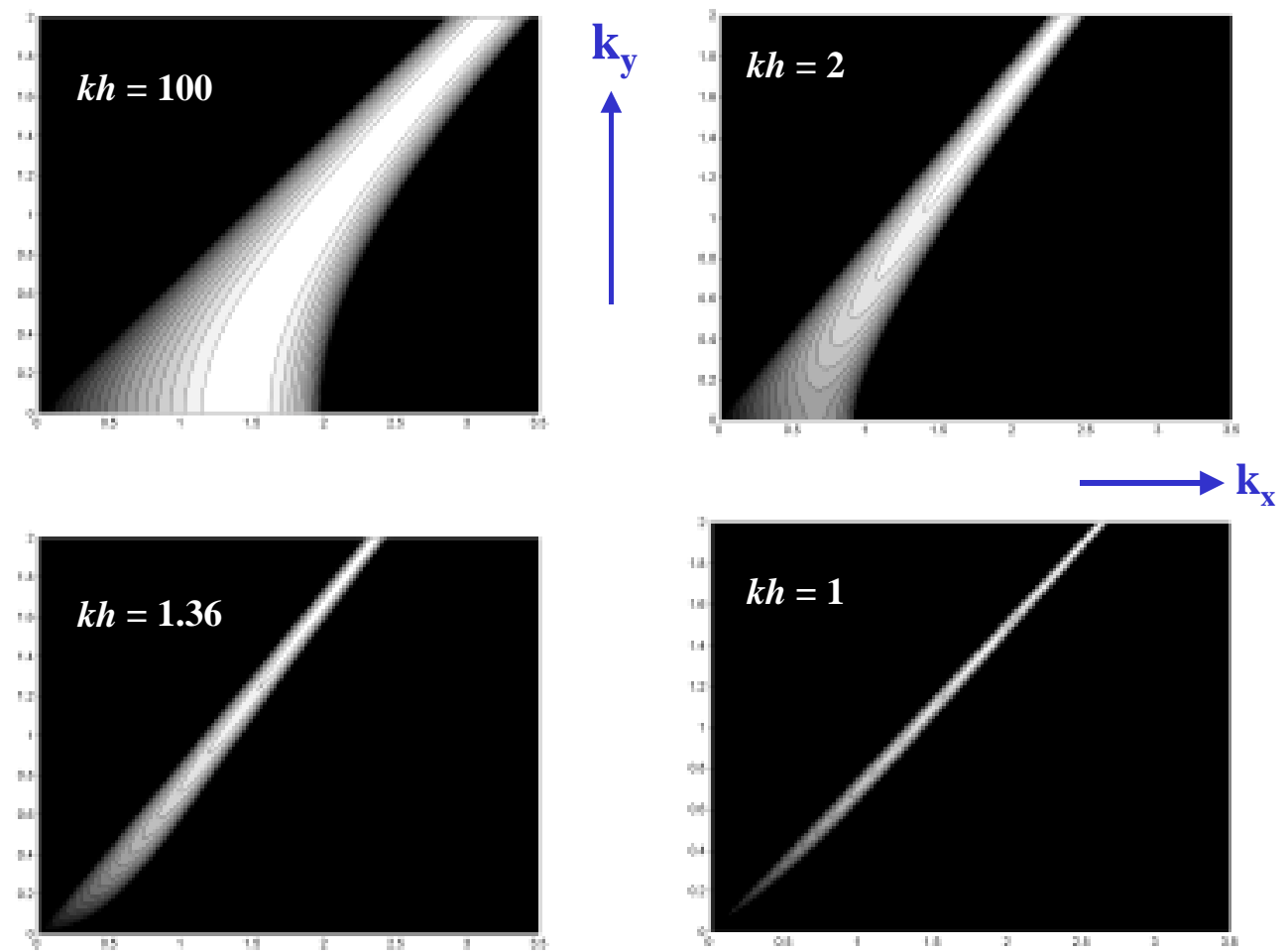

Figure 5.8. Stability diagrams in the plane of envelope wave numbers

\subsection{Extended nonlinear models of extreme wave packets}

The rogue wave phenomenon was discussed above in the framework of weakly nonlinear models. In fact, rogue waves have large amplitudes and short duration, so the approximations of weak nonlinearity and narrow-banded spectrum do not correspond exactly to real data. For instance, the nonlinear Schrödinger equation (and the Davey-Stewartson system) is symmetrical with respect to the coordinates and this leads to symmetrical waveforms in the evolution process (if it was initially symmetrical). Laboratory experiments show the asymmetry of the wave envelope for large amplitudes; see, for instance, Shemer et al. (1998). Weakly nonlinear models predict also incorrect values for the BF instability for short-scale modulation with regard to fully nonlinear computations given by Longuet-Higgins (see Dysthe, 1979). It means that the wave cannot be considered as a weakly nonlinear wave if its steepness is approximately greater than 0.1. For deep water, Dysthe (1979) derived a modified nonlinear Schrödinger equation to fourth order in wave steepness. One of the main results at this order is precisely the influence of the wave-induced mean flow. This equation is called now the Dysthe equation. Spatial versions of the 2D Dysthe equation are discussed by Lo \& Mei (1985) and Kit \& Shemer (2002). Modifications of the nonlinear Schrödinger equation result in significant reducing of the BF instability region, and the Stokes wave will be stable with respect to short-scale disturbances according to the predictions of rigorous theories. Modified nonlinear Schrödinger equations 
describe better the data of laboratory experiments (Shemer et al., 1998; Ablowitz et al., 2000, 2001).

Later, Trulsen and Dysthe (1996) extended the Dysthe equation by including higher-order linear dispersive terms (up to the fifth derivative of the wave amplitude) describing broader bandwidth water waves. Trulsen et al. (2000) noticing the importance of linear dispersion improved the Dysthe equation with exact linear dispersion. For numerical simulations of ocean waves, one must emphasize that this model does not suffer from energy leakage. The extended Dysthe equation is written as follows

$$
i\left(\frac{\partial A}{\partial t}+L\left(\partial_{x}, \partial_{y}\right) A\right)=\frac{\omega_{0} k_{0}^{2}}{2}|A|^{2} A-\frac{i \omega_{0} k_{0}}{4}\left(6|A|^{2} \frac{\partial A}{\partial x}+A^{2} \frac{\partial A^{*}}{\partial x}\right)+k_{0} A \frac{\partial \Phi(z=0)}{\partial x},
$$

where $\Phi(x, z, t)$ is the induced mean flow satisfying the Laplace equation

$$
\Delta \Phi=0
$$

with the boundary conditions on sea surface $(z=0)$

$$
\frac{\partial \Phi}{\partial z}=\frac{\omega_{0}}{2} \frac{\partial|A|^{2}}{\partial x}
$$

it vanishes far from the sea surface (in deep water). The pseudo-differential operator L can be obtained from the Fourier integral with the kernel $\omega(k)-\omega\left(k_{0}\right)$, it is

$$
L\left(\partial_{x}, \partial_{y}\right)=i\left[\left(1-i \partial_{x}\right)^{2}-\partial_{y}^{2}\right]^{1 / 4}-i
$$

Additional terms to the nonlinear Schrodinger equation transform initial Gaussian shape of the spectrum in asymmetric profile with a steepening of the low-frequency side providing a downshift of the spectral peak (Dysthe et al., 2003). The new principal result here is the chaotic dynamics of the wave field even for regular initial data (Ablowitz et al., 2000; Ablowitz et al., 2001; Calini and Schober, 2002); so rogue waves appear and disappear randomly (the same effect is also observed in discrete computed models of the nonlinear Schrödinger equation, see Herbst and Ablowitz, 1989; Ablowitz et al., 1993). Trulsen (2001) used the extended Dysthe equation to simulate numerically the "New Year Wave" event described in section 2. 
The 2D Dysthe equation and its extensions were used to analyze random wave field evolutions. As a result, an anisotropy in the wave field behaviour is predicted. In particular, the spectrum develops asymmetrically with a downshift of the spectral peak and an angular widening; equilibrium interval, $k^{-2.5}$ is observed (Dysthe et al., 2003). The wave process is Gaussian in average only; its kurtosis oscillates around 3 (value for Gaussian distribution function), sometimes becoming very high (Onorato et al., 2002). Large values of the kurtosis correspond to large tails of the distribution function, providing higher probability of freak wave occurrence.

A more general model for fully linear dispersive and weakly nonlinear waves is based on the Zakharov equation (1968), which can give more accurate expressions for nonlinear dispersive terms. Using the Hamiltonian formalism, Zakharov (1968) derived the appropriated integral equations for water wave field. Firstly, the standard potential equations of the motion are transformed as follows

$$
i \frac{\partial b(\vec{k})}{\partial t}=\frac{\delta H}{\delta b^{*}(\vec{k})},
$$

where $b(\vec{k})$ is the complex amplitude and $H\left(b, b^{*}\right)$ is the Hamiltonian. The asterisk means complex conjugate. In the spectral space the complex Fourier amplitudes $b(\vec{k})$ are expressed by means of integral power series in Fourier amplitudes of the elevation of the free surface and the velocity potential at the free surface. Then the Hamiltonian $H$ is developed in terms of an integral power series in the complex amplitudes $b(\vec{k})$

$$
H=\int \omega(\vec{k}) b(\vec{k}) b^{*}(\vec{k}) d \vec{k}+\sum_{n=4}^{\infty} H_{n},
$$

$H_{n}$ are of power $n$ in $b(\vec{k})$ responsible of nonlinear effects (for deep water). By taking into account $H_{4}$ and $H_{5}$ in the truncated Hamiltonian we arrive at the so-called five-wave reduced Zakharov equations derived by Krasitskii (1994)

$$
\begin{gathered}
i \frac{\partial b_{0}}{\partial t}=\omega_{0} b_{0}+\int V_{0123} b_{1}^{*} b_{2} b_{3} \delta_{0+1-2-3} d \vec{k}_{1} d \vec{k}_{2} d \vec{k}_{3}+ \\
+\int W_{01234} b_{1}^{*} b_{2} b_{3} b_{4} \delta_{0+1-2-3-4} d \vec{k}_{1} d \vec{k}_{2} d \vec{k}_{3} d \vec{k}_{4}+\frac{3}{2} \int W_{43210} b_{1}^{*} b_{2} b_{3} b_{4} \delta_{0+1+2-3-4} d \vec{k}_{1} d \vec{k}_{2} d \vec{k}_{3} d \vec{k}_{4},
\end{gathered}
$$


where $b_{0}=b(\vec{k}), b_{j}=b\left(\vec{k}_{j}\right), \omega_{0}=\omega(\vec{k}), V$ and $W$ are functions of wave vectors: $\vec{k}, \vec{k}_{1}$ and so on, $\delta$ is the Kroneker symbol. Kernels $V$ and $W$ are satisfying symmetry conditions. This equation describes four-wave and five-wave resonant interactions. Stiassnie and Shemer (1984) extended the derivation, including five-wave interaction, to water waves on finite depth. Note nevertheless that their kernels lacked these symmetry properties. Within the framework of fourwave interaction, equation (5.16) reduces to the nonlinear Schrödinger equation under the assumption of narrow-banded gravity waves. In finite depth one can recover the DaveyStewartson system. While the Zakharov equation is the most sophisticated approximate model for the spatio-temporal evolution of water waves it has not the ease of use of the nonlinear Schrödinger equation. Note also that the spatial version of the Zakharov equation (Shemer et al., 2001,2002 ) is very convenient for the processing of water wave experiments in $2 \mathrm{D}$ wave tanks.

Annenkov \& Badulin (2001) selected in the frequency spectrum of a 20 minutes wave (New Year Wave) recording at the 'Draupner' platform (it is shown in Figure 2.3), the specific component peculiar to five-wave interactions. This component corresponds to class II instabilities phase-locked to the dominant component of the spectrum. Physically this instability is known to generate water wave horseshoe patterns frequently observed on the sea surface. More details can be found in the review paper of Dias and Kharif (1999). In order to have a better understanding of the role of this kind of resonance in the formation of rogue waves Annekov \& Badulin (2001) performed numerical simulations of equation (5.16) in which modulational and five-wave interactions are both taken into account. They showed that cooperative effects of these interactions may be responsible for the occurrence of rogue waves and emphasize the role of oblique waves in this process.

The Zakharov equation should be a powerful model to study the formation of rogue waves, which are not due only to modulational instabilities. The paper by Annekov and Badulin (2001) seems to be a first tentative in this direction, where the authors apply this equation to investigate rogue waves.

\subsection{Weakly nonlinear rogue waves in shallow water}

For shallow water the ratio of nonlinearity to dispersion is usually high and the generation of the highest harmonics become more effective. The approximation of the modulated quasimonochromatic wave used in previous section is valid only for very weak waves. The simplified model of 2D unidirectional waves in the shallow water taking into account weak nonlinearity and dispersion is the Korteweg - de Vries equation 


$$
\frac{\partial \eta}{\partial t}+c\left(1+\frac{3 \eta}{2 h}\right) \frac{\partial \eta}{\partial x}+\frac{c h^{2}}{6} \frac{\partial^{3} \eta}{\partial x^{3}}=0
$$

where $c=(g h)^{1 / 2}$ is long wave speed. Derived in 1895 this equation was the first to exhibit exact solutions of the Cauchy problem by using the inverse scattering approach. The solutions of (5.17) are stable (see Figure 5.8 for comparison), and, therefore, the nonlinear mechanism of the rogue wave formation due to modulation instability does not "work" in shallow water. If the initial wave field presents weakly modulated wave train, its form is modified in the process of the wave evolution, but the wave amplitude does not vary significantly (Kit et al., 2000). Thus, the rogue wave can appear only due to focusing mechanism.

To demonstrate the nonlinear - dispersive focusing, the solution of the Cauchy problem for initial singular data (delta-function) can be used. According to the exact solution, the delta function evolves into the solitary wave (soliton) and oscillating dispersive tail located in space according to the values of the speed of each component (the soliton moves with a larger speed and is in front of the wave train). Due to invariance of the Korteweg - de Vries equation with respect to the reversal of time and coordinate, this wave field inverted in space should transform into the initial disturbance at fixed time, and then again transforms into a soliton and a dispersive tail. It means that there is no principal limitation for the formation of abnormal waves of large amplitude. Therefore, the wave focusing mechanism is applicable in nonlinear case also but the wave field structure is more complicated, including solitons and amplitude-frequency modulated wave packets. This process was investigated in details in papers (Pelinovsky et al., 2000; Kharif et al., 2000) and shown in Figure 5.9 (in the system of coordinates moving with the speed, $c$ ). The value of the peak of the wave field in the domain increases rapidly and then rapidly decreases (Figure 5.10), and this explains the rare and "short-lived" character of the freak wave. It is important to mention that the freak wave in the framework of the Korteweg - de Vries equation is due mainly to frequency modulated dispersive wave train, not by solitons. Solitary waves do not attract to each other in the process of their interaction and cannot focus in a single point. Moreover, from the direct solution of the Cauchy problem it follows that initial large positive pulse will evolve in a small-amplitude wave field (involving solitons), only if the effective Ursell number of the initial disturbance $\left(U r=A_{0} \lambda_{0} / h^{3}\right)$ is small enough (Pelinovsky et al., 2000). For opposite case, generated solitons will have large amplitudes compared with initial amplitude, and the amplitude criterion for the freak wave event will not be satisfied. It means that the freak wave on shallow water is an almost linear wave in spite of its large amplitude. If the initial disturbance is negative (the hole on sea surface), it evolves into damped wave train only (with no solitons), and there is no limitation on the Ursell number of negative freak wave. 
Solutions of periodic problems for the Korteweg-de Vries equation are more complicated. First of all, the singular solution (called positon) should be mentioned (Matveev, 2002)

$$
\frac{\eta}{h}=-128 \kappa^{2} \frac{\sin \Theta(\sin \Theta-\kappa \Psi \cos \Theta)}{(\sin 2 \Theta-2 \kappa \Psi)^{2}},
$$

where

$$
\Theta=\frac{\sqrt{6} \kappa}{h}\left(x-\left(1-4 \kappa^{2}\right) c t\right), \quad \Psi=\frac{\sqrt{6}}{h}\left(x-\left(1-12 \kappa^{2}\right) c t\right),
$$

are determined by the one parameter, $\kappa$. The positon has the same behaviour that the soliton: elastic collision, shape conservation and so on. A positon solution as a function of $x$ has a second-order pole and, therefore, has an infinite energy. Such solutions cannot be realized physically. They show moreover a tendency of smooth solutions of the Korteweg-de Vries equation to be close to waves with very high peaks. As Matveev (2002) pointed out, the proper nonsingular regularization is presumably provided by finite-gap quasi-periodic solutions of the Korteweg-de Vries equation corresponding to spectra with very narrow forbidden zones and very narrow spectral bands between these zones in associated spectral problem.

The detailed analysis of the periodic solutions of the Korteweg-de Vries equation expressed through the theta-functions is given in series of papers by Osborne and co-authors; see for instance, Osborne, 1995, and Osborne et al., 1998. His approach can be called the nonlinear Fourier method: the solution of the Korteweg-de Vries equation is represented by a linear superposition of nonlinear oscillatory modes (multi quasi-cnoidal waves) in the associated spectral problem. The number of such modes is not too large as in the classic Fourier method, and this leads to an effective analysis of the wave field (including random sea state) and selection of soliton components. The freak wave in this approach is the superposition of these modes with suitable phases. Numerical experiments with linear superposition of strong random and weak frequency modulated deterministic components were performed by Pelinovsky et al. (2000). Figure 5.11 displays the formation of the freak wave from this quasi-random state. 


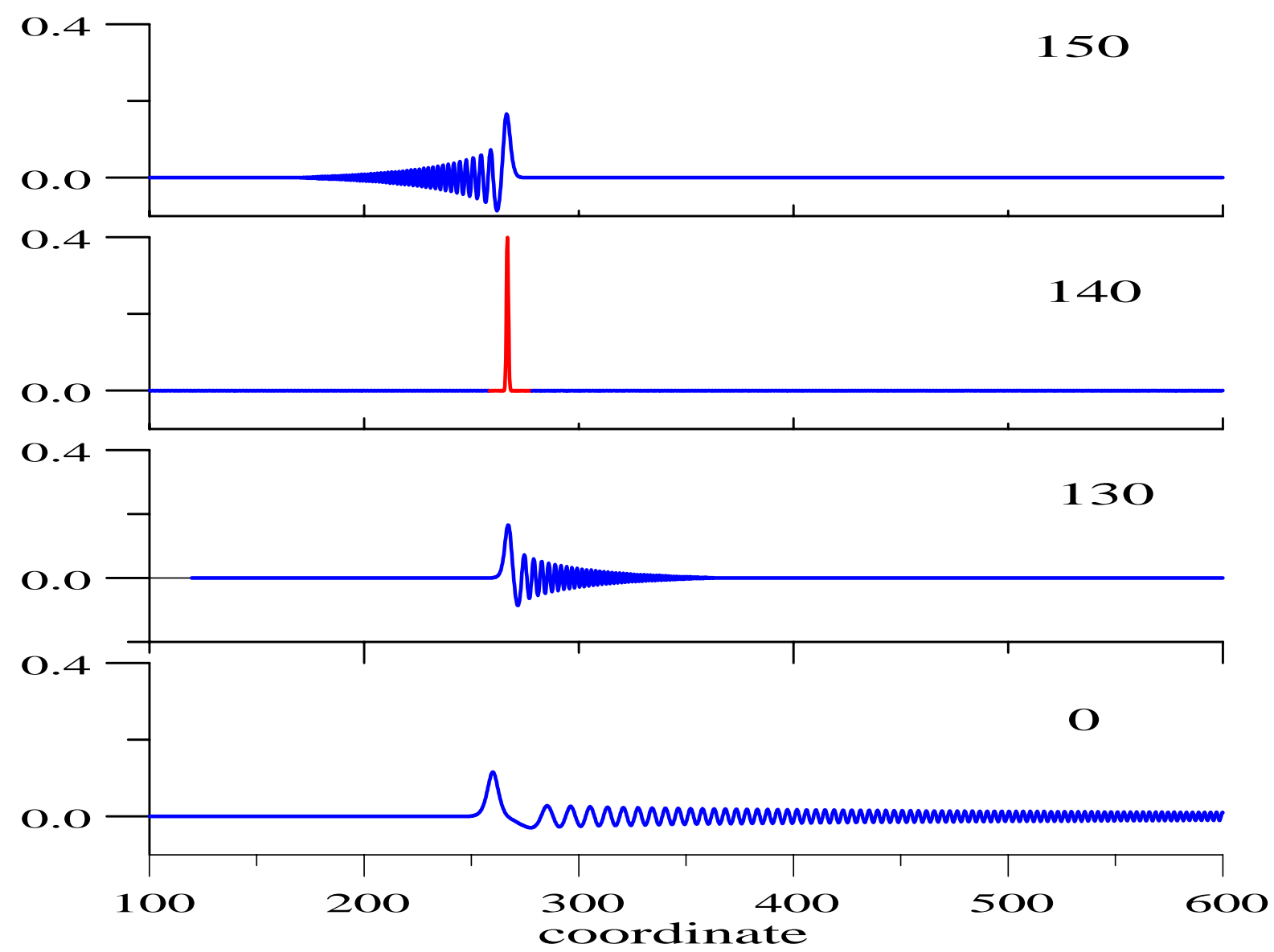

Figure 5.9. Freak wave formation in shallow water

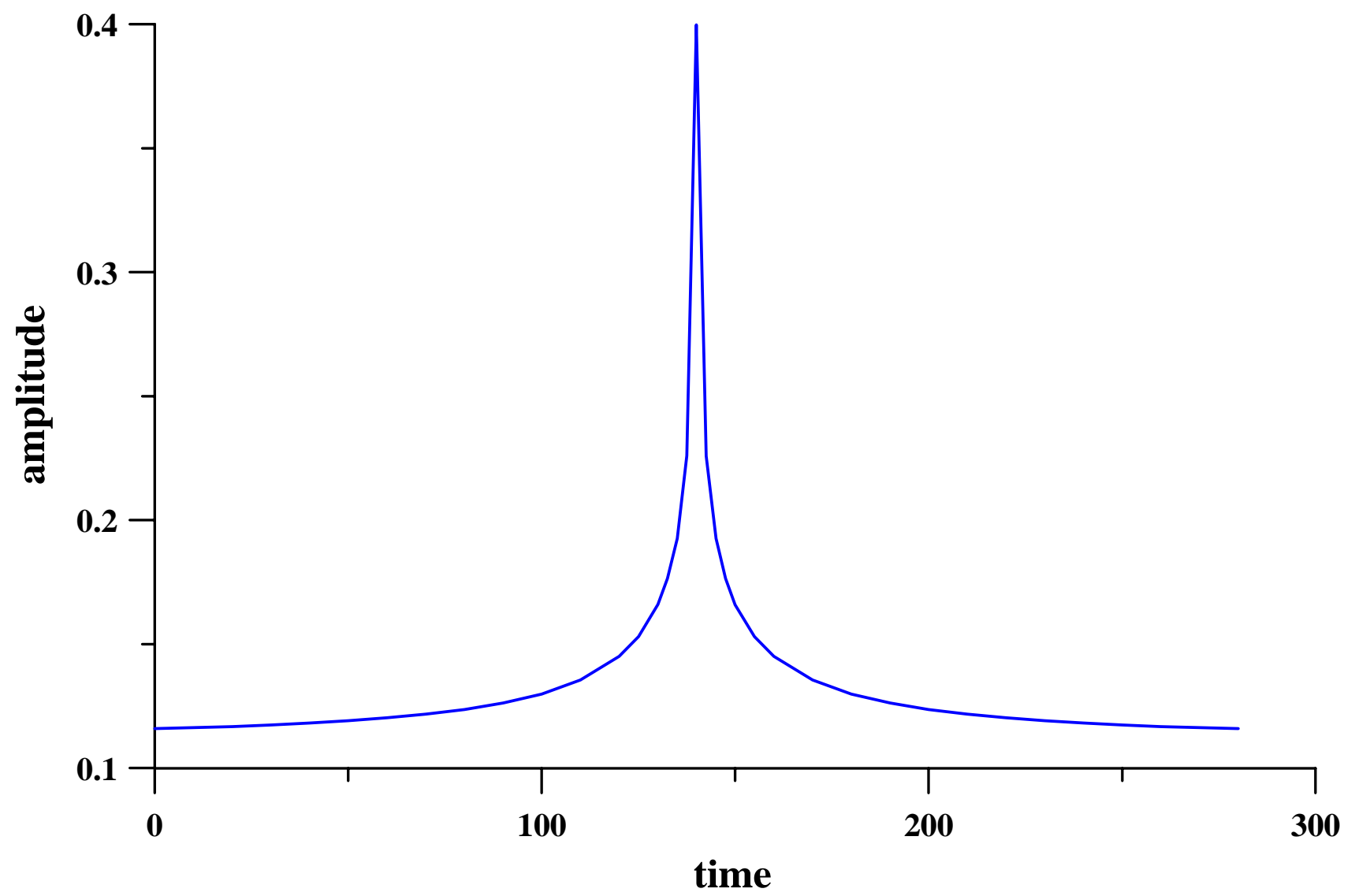

Figure 5.10. Maximum wave amplitude versus time in the process of the freak wave formation 
The present analysis shows that the wave focusing should be the main mechanism of the rogue waves in shallow water. But recently, Onorato et al. (2003), studying the nonlinear interactions of waves with a doubled-peaked power spectrum in shallow water, derived the coupled nonlinear Schrödinger equations and showed that uniform wave train can be unstable to small perturbations. It means that in the case of doubled-peaked spectrum the modulational instability will lead to the freak wave formation as for deep water, but this process has not yet been investigated.

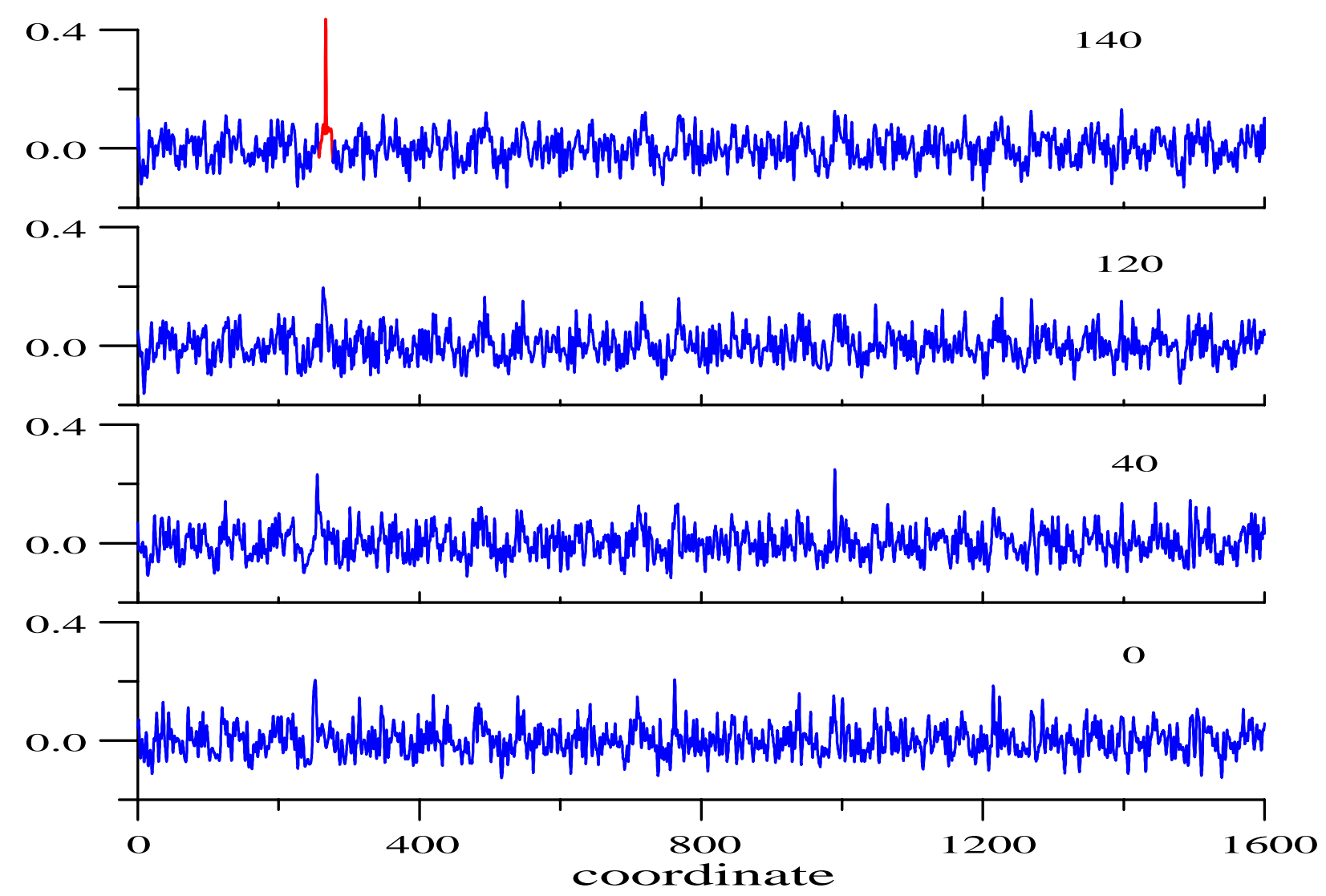

Figure 5.11. Freak wave formation from a random field

The 3D wave field in shallow water contains solitons and wave trains propagating in different directions. The soliton behaviour in space has the non-trivial feature of the resonant interaction as for periodic waves. To demonstrate this the weakly two-dimensional analogue of the Korteweg-de Vries equation can be considered, it is famous Kadomtsev-Petviashvili equation

$$
\frac{\partial}{\partial x}\left[\frac{\partial \eta}{\partial t}+c\left(1+\frac{3 \eta}{2 h}\right) \frac{\partial \eta}{\partial x}+\frac{c h^{2}}{6} \frac{\partial^{3} \eta}{\partial x^{3}}\right]=-\frac{c}{2} \frac{\partial^{2} \eta}{\partial y^{2}}
$$


The equation (5.19) is integrable as the Korteweg - de Vries and nonlinear Schrödinger equations. In particular, the two-soliton solution can be written explicitly (in the system of coordinates moving with long-wave speed), see Satsuma (1976), Onkuma \& Wadati (1983) and Pelinovsky (1996)

$$
\begin{gathered}
\eta(x, y, t)=\frac{4 h^{3}}{3} \frac{\partial^{2} \ln F}{\partial x^{2}}, \quad F(x, y, t)=1+\exp \varsigma_{1}+\exp \varsigma_{2}+d \exp \left(\varsigma_{1}+\varsigma_{2}\right), \\
\boldsymbol{\zeta}_{i}=k_{i} x-p_{i} y-V_{i} t, \quad V_{i}=c\left(k_{i}^{2}+3 p_{i}^{2}\right), \quad d=\frac{\left(k_{1}+k_{2}\right)^{2}-\left(p_{1}-p_{2}\right)^{2}}{\left(k_{1}-k_{2}\right)^{2}-\left(p_{1}-p_{2}\right)^{2}} .
\end{gathered}
$$

One interesting example of the applicability of the two-soliton solution (5.20) is the analysis of oblique soliton approaching to a vertical wall $(y=0)$. The boundary conditions on the wall is satisfied automatically, if the soliton amplitudes (proportional to $k$ ) and speeds $(V)$ are equal, and $\mathrm{p}_{1}=-\mathrm{p}_{2}$ (full analogue of the Snell law for reflection of oblique waves). Omitting the mathematical manipulations, the wave height on the wall is expressed as

$$
\frac{H}{H_{0}}=\frac{4}{1+\sqrt{1-\frac{3 H_{0}}{4 h \tan ^{2} \theta}}},
$$

where $H_{0}$ is the amplitude of the incident soliton and $\theta$ is the angle between the soliton front and the $y$-axis. When the angle is small (almost along wall propagation) and closed to the characteristic nonlinear parameter $\left(H_{0} / h\right)$, the wave amplification can be very significant (by a factor 4!) to compare with normal approaching, when the wave height on the wall is almost twice greater than the soliton amplitude. The significant amplification of the soliton amplitude near the wall firstly was found by Miles (1977) using a perturbation technique in the framework of the Boussinesq equation. For very small angles of the incident wave, the solution (5.21) is not valid and the wave propagation is not stationary. In this case, the soliton interaction induces a "virtual" soliton (the "Mach stem"). The resonance processes in the soliton interaction are studied intensively, and recently Peterson et al. (2003) suggested that multi-soliton solutions of the Kadomtsev-Petviashvili equation can explain 3D extreme wave events in shallow water (not in the near-wall area only). The shapes of the large-amplitude wave occuring in the process of the two-soliton interaction for various angles between soliton fronts are given in Figure 5.12 from 
the paper by Peterson et al. (2003). It is important to note that such a wave has infinite "lifetime" and propagates with constant speed. Specific numerical simulations should be performed to evaluate the importance of these interactions on the background of random wave fields.

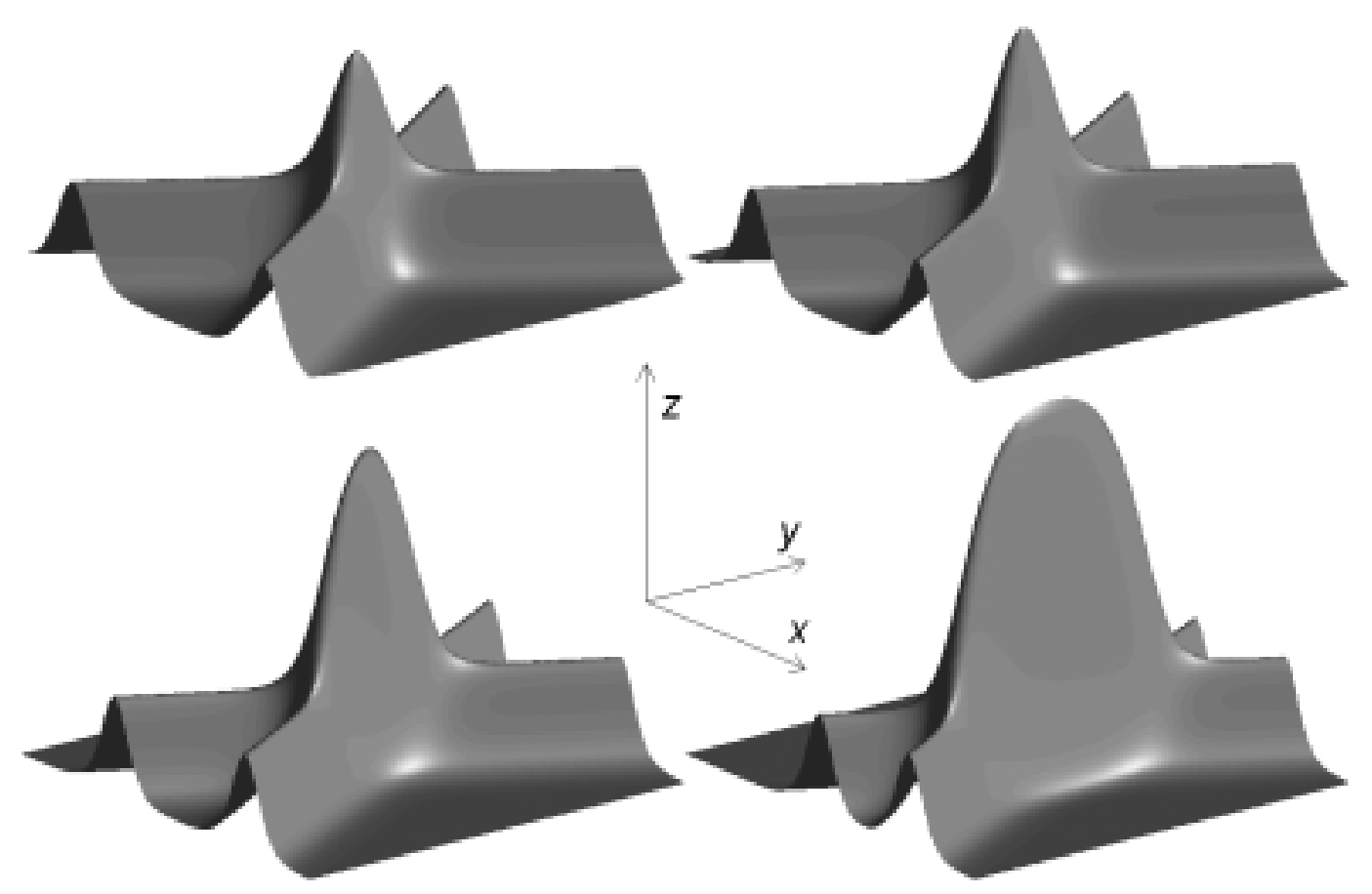

Figure 5.12. Large-amplitude waves occuring in the process of soliton interaction

\subsection{Fully nonlinear models of the extreme waves}

We have discussed the rogue wave phenomenon within the framework of weakly nonlinear models. In fact the rogue waves are really huge waves, which should be considered as strongly nonlinear waves. Studies about rogue waves within the framework of fully nonlinear and dispersive models are very few. The governing equations are the Laplace equation for the velocity potential $\phi(x, y, z, t)$ with fully nonlinear dynamic and kinematic boundary conditions at the free surface $z=\eta(x, y, t)$

$$
\Delta \phi=0 \quad \text { for } \quad-h \leq z \leq \eta(x, y, t)
$$

with kinematic and dynamic boundary conditions on the sea surface, $z=\eta(x, y, t)$

$$
\frac{\partial \eta}{\partial t}+\frac{\partial \phi}{\partial x} \frac{\partial \eta}{\partial x}+\frac{\partial \phi}{\partial y} \frac{\partial \eta}{\partial y}-\frac{\partial \phi}{\partial z}=0
$$




$$
\frac{\partial \phi}{\partial t}+\frac{1}{2}|\nabla \phi|^{2}+g \eta=0
$$

and the boundary condition on sea bottom $(z=-h)$

$$
\frac{\partial \phi}{\partial z}=0
$$

For infinite depth the condition (5.25) becomes $\nabla \phi \rightarrow 0$ as $z \rightarrow-\infty$.

Generally direct numerical simulations of rogue waves requires to consider large spatial and temporal domains and need to use very large numbers of nodes and time steps. Henderson et al. (1999) investigated numerically the time evolution of a two-dimensional almost uniform wave train with a small growing modulation. They performed numerical experiments: it was observed that the energy focusses into a short group of steep waves called steep wave event (SWE). Details about the numerical code they used to study water wave modulations can be found in the paper by Dold (1992). They found that the breather solutions of the nonlinear Schrödinger equation fit numerical SWE rather well. These SWE's can be considered as rogue wave events. So, the rogue wave mechanism related with the BF instability is confirmed in fully nonlinear computations. Figure 5.13 concerns the water wave profile, at the maximum of modulation, of an initially Stokes waves of wave steepness 0.13 disturbed by its most unstable perturbation (Skandrani, 1997). One can observe the formation of the huge wave due to the modulational instability. One can observe also a big hole. Note that this direct numerical simulation performed by using the Dommermuth \& Yue (1987) method, breaks down after few modulationdemodulation cycles owing to possible local breaking phenomena (see Dias \& Kharif, 1999).
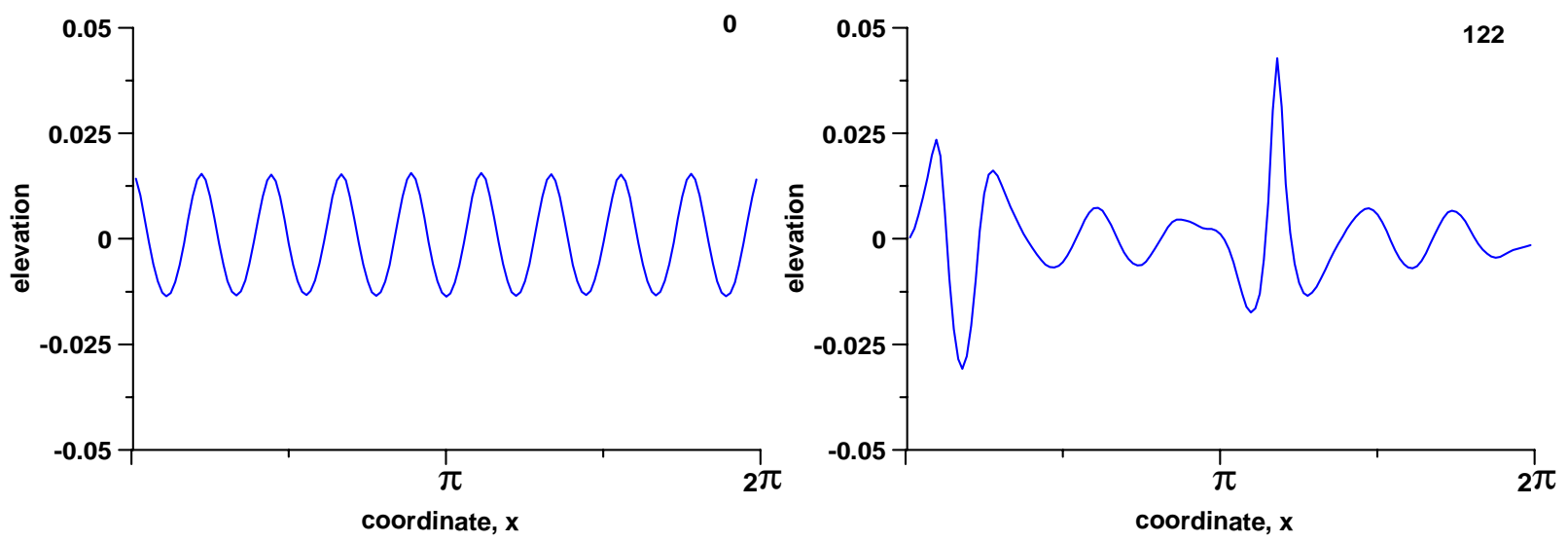

Fig. 5.13 Water wave profile (left) at $\mathrm{t}=0$, and (right) at the maximum of modulation (numbers - time normalized by the fundamental wave period), see Skandrani (1997) 
Very recently Grue (2002), Clamond \& Grue (2002) and Clamond et al. (2003) performed a fully nonlinear numerical simulation of the long time evolution of a two-dimensional localized long wave packet (steepness 0.091) and compared their computations with those resulting from the classic nonlinear Schrödinger and extended Dysthe equations. Their computations were obtained by using the fast computational method for nonlinear water waves developed previously by Clamond \& Grue (2001) and were consistent with those provided by the pseudo-spectral method of Dommermuth \& Yue (1987). The potential is written in a finite perturbation series up to given order $M$, which is the order of approximation. For instance, results obtained with $M=4$ correspond to the Zakharov equation or to the Dysthe equation when the spectrum is narrowbanded. Figure 5.14 shows the comparison for two values of $M ; M=4$ and $M=8$ (Clamond et al., 2003). Clamond et al. (2003) found, as expected, that there is a good agreement with approximate models for relatively short period of time, $O\left(T_{0} / \mathcal{E}^{2}\right)$, for the nonlinear Schrödinger equation and $O\left(T_{0} / \varepsilon^{3}\right)$ for the Dysthe equation, where $T_{0}$ is the fundamental wave period and $\varepsilon$ is the wave steepness). For long periods of time it is found that approximate models should not necessarily provide correctly the nonlinear dynamics of the water waves and subsequently the probability of occurence of rogue waves. This conclusion applies also for the Zakharov equation that is the more accurate among all the approximate models. The time evolution of the maximum crest amplitude is shown in Figure 5.15. For large time the fully nonlinear model shows the absence of the rogue waves meanwhile the extended Dysthe and Zakharov equations predict their appearance regularly. Of course, this is only one example of long-time evolution of isolated wave packets, but it emphasizes that full nonlinearity plays an important role for very long-time simulations. Bateman et al. (2001) extended the spectral wave model of Craig \& Sulem (1993) to describe the water waves evolution of extreme waves due to the focusing of wave components involving spread of energy in both frequency and direction. Occurrence of rogue waves would be due to direction spreading of waves. Using a JONSWAP spectrum, a three-dimensional simulation of the formation of a rogue wave is presented. Within the framework of fully nonlinear equations Brandini and Grilli (2001) investigated numerically the formation of rogue waves by using both spectral methods and a three-dimensional boundary element method.

The previous deterministic numerical experiments were performed to better understand the physical mechanisms that can contribute to the formation of abnormal waves. Mori \& Yasuda (2002) considered exact equations numerically integrated by using the pseudo-spectral method of Dommermuth \& Yue (1987) to investigate wave statistics. Unfortunately, they truncated the solution to fourth-order in wave steepness and this corresponds to the Zakharov equation. So their model does not capture the full nonlinearity contained in the equations. This limitation is all the more serious, as the water is shallow. As noted previously for long-time computation the 
water wave evolution can be very different from that given by fully nonlinear models. However, one believes that their conclusions remain valid when higher-order nonlinear interactions are considered, that is nonlinear terms should affect the long-term statistics of the waves. They emphasized that nonlinear interactions increase the occurrence probability of rogue waves in deep water in comparison with the Rayleigh distribution.
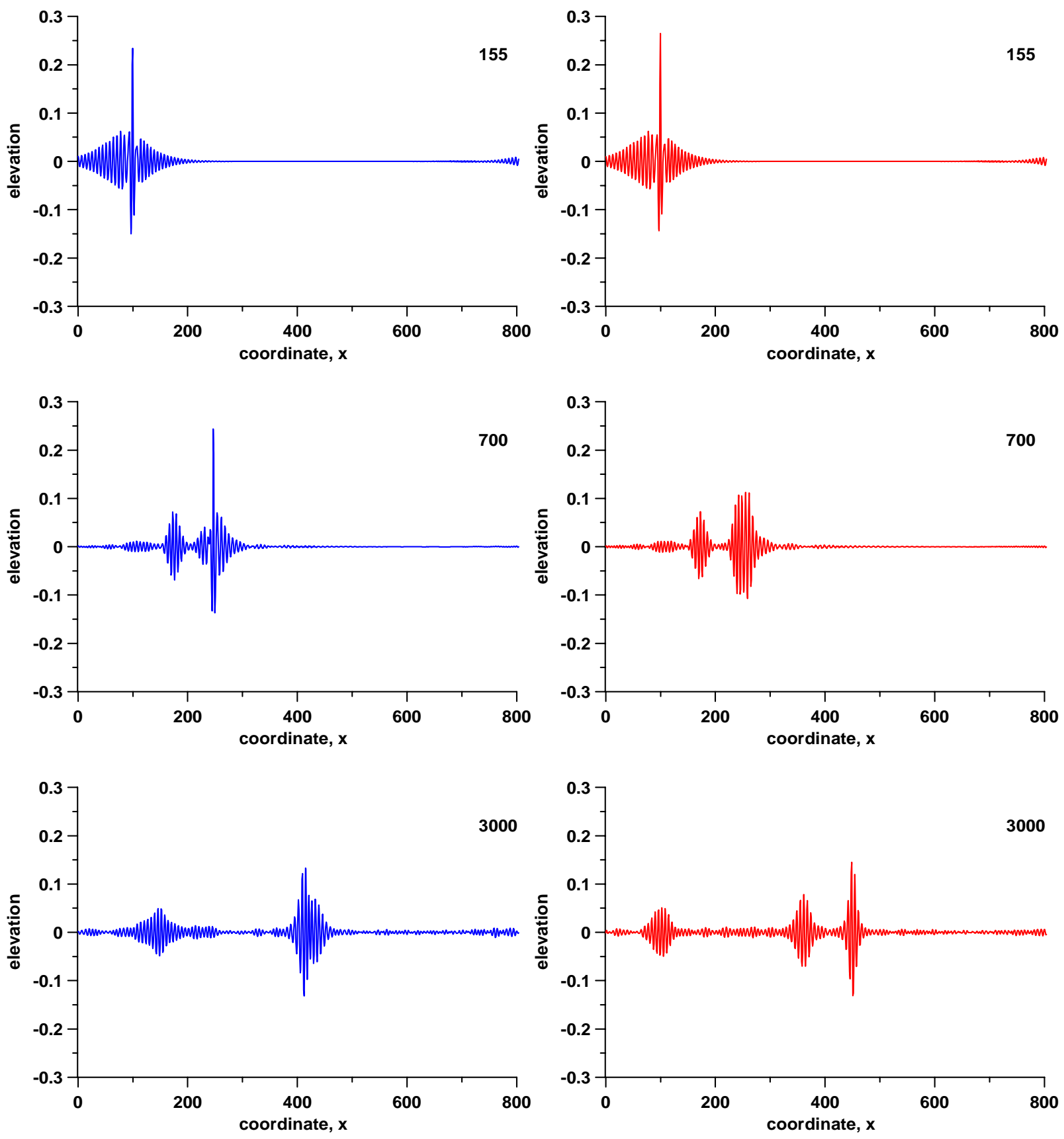

Fig. 5.14. Wave packet evolution in the framework of the fully nonlinear equations with $M=$ 4 (left) and $M=8$ (right)

Recently, Zakharov et al. (2002) proposed a new method for numerical simulation of fully nonlinear waves based on combination of the conformal mapping and Fourier transform. The total number of harmonics was 12288 . Choosing the initial condition as the superposition of the 
Stokes wave with steepness 0.1 and weak Gaussian random field, they obtain the formation of the freak wave with the amplitude exceeding the initial level more than three times.

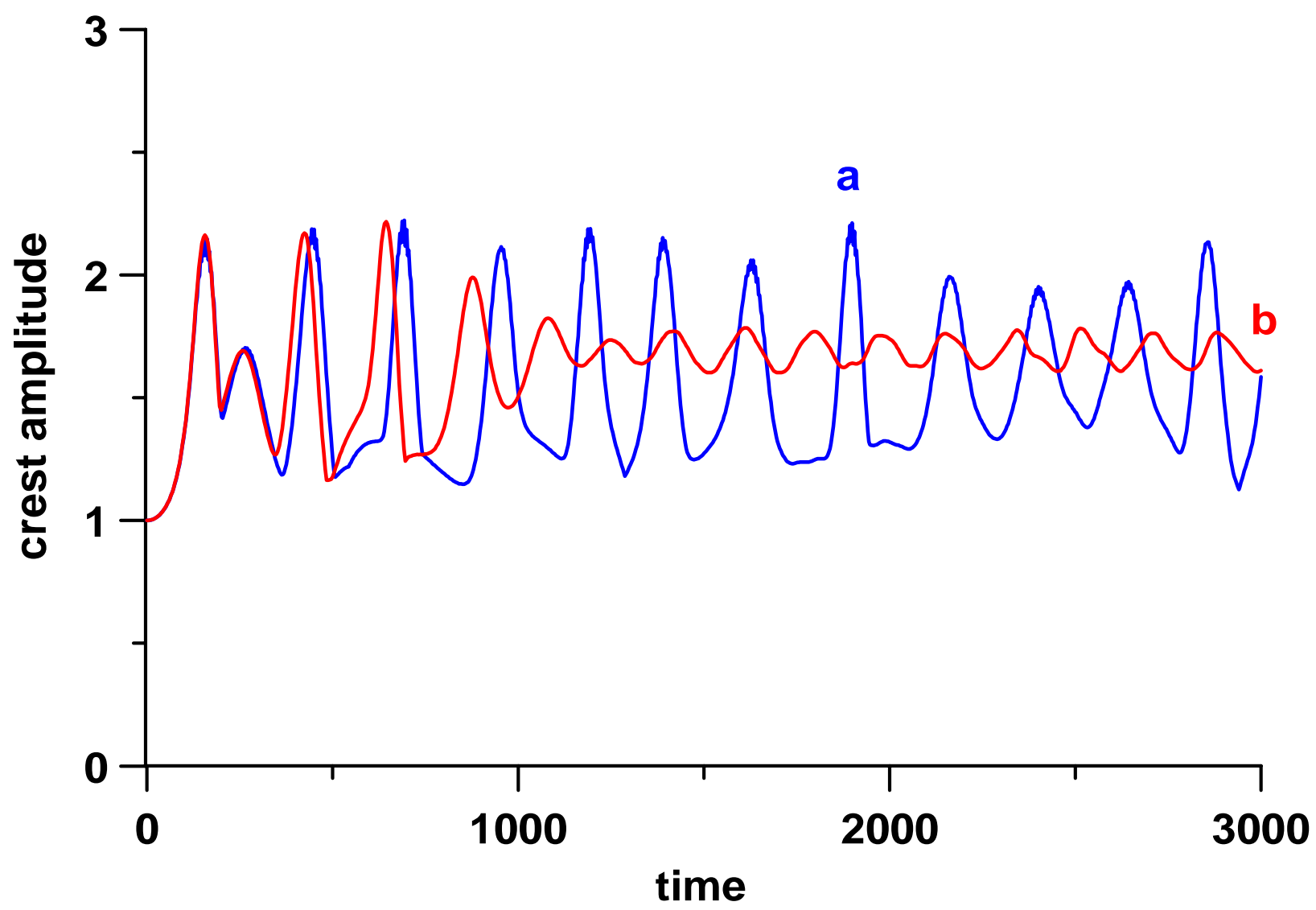

Fig. 5.15. Time evolution of the maximum crest amplitude (normalized by initial amplitude) computed in the framework of the fully nonlinear equations with (a) $M=4$ and (b) $M=8$ (from Clamond et al., 2003)

In ocean engineering the safe and economic design of any fixed or floating structures depend on a reliable estimate of the extreme wave loading. The knowledge of the underlying wave particle kinematics beneath the largest wave crests represents key information appropriate to the determination of the design loading (Smith \& Swan, 2002). Johannessen \& Swan (1997) showed that water particle kinematics is strongly dependent upon the nonlinear wave-wave interaction. They emphasized that if an appropriate description of a extreme wave event is to be achieved, both nonlinearity and unsteadiness of the wave motion must be incorporated. Bateman et al. (2003) computed velocities and accelerations beneath extreme three-dimensional ocean wave. They confirmed the result of Longuet-Higgins (1986) that the maximum magnitude of the vertical acceleration arises at some distance beneath the water surface. 


\subsection{Nonlinear models of the wave-current interaction and atmospheric forcing}

Smith (1976) is the first who developed a nonlinear model to investigate the giant waves occurence in the Agulhas Current. He derived a 2D nonlinear Schrodinger equation with an additional term $k(\partial U \partial x) A$ in the vicinity of the blocking point (4.30); for 2D wave propagating against an opposite current it is

$$
i \frac{\partial A}{\partial t}=\frac{\omega}{8 k^{2}} \frac{\partial^{2} A}{\partial x^{2}}+\frac{\omega k^{2}}{2}|A|^{2} A-k\left|\frac{d U}{d x}\right| x A
$$

where $d U / d x$ is calculated at the blocking point, and the wave frequency, $\omega_{0}$, and wave number, $\mathrm{k}_{0}$ satisfy the dispersion relation (4.29) at the blocking point. The last term in (5.26) can be easily obtained from the linear dispersion relation taking into account the Doppler shift of the wave frequency. If nonlinearity is negligible, equation (5.26) coincides with the Airy equation (for monochromatic wave) and describes the wave field on caustics; see (4.33) and (4.34). In the nonlinear case the solution of (5.26) is the Painleve transcendents of the second kind and qualitatively it resembles to the Airy function with transition from sinusoidal to exponential behaviour slightly displaced from the blocking point; see Smith (1976) for details. Therefore, nonlinearity does not change the scenario of the wave blocking on opposite current. Also, Smith (1976) observed that the giant wave should be asymmetric.

In reality, the situation becomes more complicated if a wide spectrum for water waves is considered. In particular, the equation (5.25) has the following soliton solution (Chen and Liu, 1976)

$$
\begin{gathered}
A(x, t)=B \operatorname{sech}\left[\sqrt{2} k B\left(x-B \omega t / \sqrt{8}+\omega|d U / d x| t^{2} / 8 k\right) \mid \times\right. \\
\times \exp \left\lfloor i\left(\sqrt{2} k^{2} B x+\omega k B t^{2}|d U / d x| / \sqrt{32}-\omega t^{3}|d U / d x|^{2} / 24\right)\right] .
\end{gathered}
$$

This soliton moves with the variable speed

$$
V=\frac{\omega B}{\sqrt{8}}-\frac{\omega}{4 k}\left|\frac{d U}{d x}\right| t
$$


It moves to the blocking point, penetrates the blocking point and then is reflected. Surprisingly, the soliton amplitude remains constant in the process of wave propagation in inhomogeneous medium due to the balance between dispersive focusing (defocusing) and attenuation (amplification) in the zone of the non-uniform current.

Moreover, the following transformation (Chen and Liu, 1976; Peregrine \& Smith, 1979)

$$
A(x, t)=A^{\prime}\left(x^{\prime}, t\right) \exp \left[i\left(k x t\left|\frac{d U}{d x}\right|+\frac{\omega t^{2}}{32}\left|\frac{d U}{d x}\right|^{2}\right)\right], \quad x^{\prime}=x+\frac{\omega t^{2}}{8 k}\left|\frac{d U}{d x}\right|
$$

gives the constant-coefficient nonlinear Schrodinger equation (5.1) for the function $A^{\prime}\left(x^{\prime}, t\right)$, and therefore, the appearance of a freak wave becomes obvious. Taking into account that the transformation (5.26) corresponds to frequency modulated wave trains, the zone of the caustics is wide compared with quasi-monochromatic waves. So, up to now the problem of the description of rogue waves on opposite current is not investigated in details.

Nonlinear models of large waves generated by random atmospheric forcing is studied mainly in the framework of the kinetic equation for the spectral intensity of wind waves (Lavrenov, 1998a,b). To study the form of the freak wave it is necessary to solve directly the nonlinear evolution equations with random forcing. Mathematically, the problem of stochastic nonlinear evolution equations has been studied (see for references the recent papers by Debussche and Menza, 2002 and Xie, 2003 where stochastic nonlinear Schrodinger and Korteweg - de Vries equations are solved), but up to now there is no link with the rogue wave phenomenon.

\section{Conclusions}

The rogue wave phenomenon has been instensively investigated these last years, theoretically and experimentally. Firstly it is important to emphasize that this phenomenon was documentally confirmed in the ocean in deep water as well as in coastal zone, and now a lot of freak wave records is collected. All the physical scenarios of possible extreme wave generation (focusing, wave-current interaction, modulational instability), in fact, were known but only now (during the last 5 years) they are "dressed" by mathematical models of various levels (linear, weakly nonlinear, fully nonlinear models). Results of numerical simulations show the behaviour of each mechanism taking into account the random character of the wind waves in the ocean. Computations provide also the probability of rogue wave occurrence for simplified conditions. The many results are very sensitive to the model parameters (shape of wave spectrum, various corrections of the weakly nonlinear evolution models, accuracy of numerical schemes for long- 
time computations). Laboratory experiments are performed mainly to study the focusing mechanism of the freak wave formation. The influence of the atmospheric forcing on the freak wave occurrence was not yet investigated. The large 3D numerical models including variable bathymetry and current field are not yet developed, and the cost of such computations is very high. Meanwhile, the progress in the understanding of the physics of the rogue wave phenomenon and development of adequate mathematical models is very significant for last years, and their results began to be use in the design of freak waves in ocean engineering.

\section{Acknowledgements}

This research is supported for EP by grants from CNRS, RFBR (02-05-65107) and INTAS (010330). 


\section{References}

Abdulaev, S.S. Chaos and dynamics of rays in waveguide media. Gordon \& Breach Sci. Publ. 1991.

Ablowitz, M.J., Schober C., and Herbst, B.M. Numerical chaos, roundoff errors, and homoclinic manifolds. Phys. Rev. Letters, 1993, vol. 71, N. 17, 2683 - 2687.

Ablowitz, M.I., Hammack, J. Henderson, D., and Schober, C.M. Modulated periodic Stokes waves in deep water. Phys. Rev. Letters, 2000, vol. 84, N. 5, 887 - 890.

Ablowitz, M.I., Hammack, J. Henderson, D., and Schober, C.M. Long-time dynamics of the modulational instability of deep water waves. Physica D, 2001, vol. 152-153, 416 - 433.

Alber, I.E. The effects of randomness of the stability of two-dimensional surface wavetrains. Proc. Roy. Soc. London, 1978, vol. A363, 525-546.

Anker, D., and Freeman, N.C. On the soliton solutions of the Davey - Stewartson equation for long waves. Proc. R. Soc. London, 1978, vol. A360, 529-540.

Annenkov, S.Yu., and Badulin, S.I. Multi-wave resonanses and formation of high-amplitude waves in the ocean. "Rogue Waves 2000" (Brest, France, 2000). Eds: M. Olagnon and G.A.Athanassoulis. Ifremer, 2001, 205 - 213.

Arnold, V.I. Singularities of caustics and wavefronts. Kluwer Acad. Publ. 1990.

Azais, J-M., and Delmas, C. Asymptotic expansions for the distribution of the maximum of Gaussian random fields. Extremes, 2002, vol. 5, 181-212.

Baldock, T.E., and Swan, C. Extreme waves in shallow and intermediate water depths. Coastal Engineering, 1996, vol. 27, 21 - 46.

Bateman, W.J.D., Swan, C., and Taylor P.H. On the efficient numerical simulation of directionally spread surface water waves. J. Comput. Physics, 2001, vol. 174, 277-305.

Bateman, W.J.D., Swan, C., and Taylor P.H. On the calculation of the water particle kinematics arising in a directionally spread wavefield. J. Comput. Physics, 2003, vol. 186, 70-92.

Boccotti, P. On the highest waves in a stationary Gaussian process. Atti Acc. Ligure, 1981, vol. 38, 271-302.

Boccotti, P. A general theory of three-dimensional wave groups. Pt 1. The formal derivation. Ocean Engineering, 1997, vol. 24, N. 3, 265-280.

Bona, J.L., and Saut, J.-C. Dispersive blowup of solutions of generalized Korteweg - de Vries equations. J. Differential Equations, 1993, vol. 103, N. 1, 3-57.

Brandini, C., and Grilli, S. Evolution of three-dimensional unsteady wave modulations. "Rogue Waves 2000” (Brest, France, 2000). Eds: M. Olagnon and G.A.Athanassoulis. Ifremer, 2001, $275-282$. 
Brown, M.G. The Maslov integral representation of slowly varying dispersive wavetrains in inhomogeneous moving media. Wave Motion, 2000, vol. 32, 247-266.

Brown, M.G. Space-time surface gravity wave caustics: structurally stable extreme wave events. Wave Motion, 2001, vol. 33, 117-143.

Brown, M.G., and Jensen, A. Experiments on focusing unidirectional water waves. J. Geophys. Research, 2001, vol. 106, N. C8, 16,917 - 16,928.

Calini, A., and Schober C.M. Homoclinic chaos increases the likelihood of rogue wave formation. Physics Letters A, 2002, vol. 298, 335-349.

Chase, G.A. Big Wave. http://bell.mma.edu/ achase/NS-221-Big-Wave.html

Chen, H.H., and Liu, C.S. Solitons in nonuniform media. Phys. Review Letters, 1976, vol. 37 , $693-697$.

Choi B.H., Pelinovsky E., Riabov I., Hong S.J. Distribution functions of tsunami wave heights. Natural Hazards, 2002, vol. 25, No. 1, 1 - 21.

Clamond, D., and Grue, J. A fast method for fully nonlinear water-wave computations. J. Fluid Mech., 2001, vol. 447, 337 - 355.

Clammond, D., and Grue, J. Interaction between envelope solitons as a model for freak wave formations. Pt. 1: Long time interaction. C.R. Mecanique, 2002, vol. 330, 575 - 580.

Clamond, D., Francius M., Grue, J., and Kharif, Ch. Strong interaction between envelope solitary surface gravity waves. 2003 , in preparation

Clauss, G. Task-related wave groups for seakeeping tests or simulation of design storm waves. Applied Ocean Research, 1999, vol. 21, 219-234.

Clauss, G. Dramas of the sea: episodic waves and their impact on offshore structures. Applied Ocean Research, 2002, vol. 24, 147 - 161.

Clauss, G., and Bergmann, J. Gaussian wave packets: a new approach to seakeeping tests of ocean structures. Applied Ocean Research, 1986, vol. 8, 190-206.

Contento, G., Codiglia, R., and D'Este, F. Nonlinear effects in 2D transient nonbreaking waves in a closed flume. Applied Ocean Research, 2001, vol. 23, 3 - 13.

Craig, W., and Sulem, C. Numerical simulation of gravity waves. J. Comput. Physics, 1993, vol. $108,73-83$.

Dias, F., and Kharif, C. Nonlinear gravity and capillary-gravity waves. Annual Review Fluid Mechanics, 1999, vol. 31, 301 - 346.

Debussche, A., and Menza, L. Numerical simulation of focusing stochastic nonlinear Schrodinger equations. Physica D, 2002, vol. 162, 131 - 154.

Dobrokhotov, S.Yu. Maslov methods in the linearized theory of gravity waves on the surface of a liquid. Soviet Physics Doklady, 1983, vol. 28, 229 - 231. 
Dobrokhotov, S.Yu., and Zhevandov, P.N. Asymptotic expansions and the Maslov canonical operator in the linear theory of water waves. 1. Main constructions and equations for surface gravity waves. Russian J. Mathematical Physics, 2003, vol. 10, N. 1, 1- 31.

Dold, J.W. An efficient surface-integral algorithm applied to unsteady gravity waves. $J$. Comput. Physics, 1992, vol. 193, 90 - 115.

Dommermuth, D.G., and Yue, D.K.P. A high-order spectral method for the study of nonlinear gravity waves. J. Fluid Mech., 1987, vol. 184, 267 - 288.

Dysthe, K.B. Note on a modification to the nonlinear Schrodinger equation for application to deep water waves. Proc. Roy. Soc. London, 1979, vol. A369, 105-114.

Dysthe, K.B., and Trulsen, K. Note on breather type solutions of the NLS as a model for freakwaves. Physica Scripta, 1999, vol. T82, 48-52.

Dysthe, K.B., Trulsen, K., Krogstad, H.E., and Socquet-Juglard, H. Evolution of a narrowband spectrum of random surface gravity waves. J. Fluid Mech., 2003, vol. 478, 1-10.

Graham, D.M. NOAA vessel swamped by rogue wave. Oceanspace, 2000, No. 284.

Grue, J. On four highly nonlinear phenomena in wave theory and marine hydrodynamics. Applied Ocean Research, 2002, vol. 24, 261 - 274.

Haver S. and Andersen O.J. Freak waves: rare realizations of a typical population or typical realizations of a rare population? Proc. $10^{\text {th }}$ ISOPE Conference, 2000, $123-130$.

Henderson, K.L., Peregrine, D.H., and Dold, J.W. Unsteady water wave modulations: fully nonlinear solutions and comparison with the nonlinear Schrodinger equation. Wave Motion, 1999, vol. 29, 34 - 361.

Herbst, B.M., and Ablowitz, M.J. Numerically induced chaos in the nonlinear Schrodinger equation. Phys. Rev. Letters, 1989, vol. 62, N. 18, 2065 - 2068.

Johannessen, T.B., and Swan, C. Nonlinear transient water waves - Pt. 1. A numerical method of computation with comparisons to 2-D laboratory data. Applied Ocean Research, 1997, vol. $19,293-308$.

Johannessen, T.B., and Swan, C. A laboratory study of the focusing of transient and directionally spread surface water waves. Proc. Royal Soc. London, 2001, vol. A457, 971 1006.

Kharif, C., Pelinovsky, E., and Talipova, T. Formation de vagues géantes en eau peu profonde. Comptes Rendus de l'Académie des Sciences, 2000, vol. 328, série IIb, N. 11, 801 - 807.

Kharif C., Pelinovsky E., Talipova T., and Slunyaev A. Focusing of nonlinear wave groups in deep water. JETP Letters, 2001, vol. 73, N. 4, 170 - 175. 
Kit, E., Shemer, L., Pelinovsky, E., Talipova, T., Eitan, O., and Jiao, H. Nonlinear wave group evolution in shallow water. J. Waterway, Port, Costal, Ocean Eng. 2000, vol. 126, N. 5, 221-228.

Kit, E., and Shemer, L. Spatial versions of the Zakharov and Dysthe equations for deep-water gravity waves. J. Fluid Mech., 2002, vol. 450, 201 - 205.

Klyatskin, V.I. Caustics in the random media. Waves in random media, 1993, vol. 3, 93-100.

Kokorina, A., and Pelinovsky, E. Applicability of the Korteweg-de Vries equation for description of the statistics of freak waves. J. Korean Society Coastal and Ocean Engineers, 2002, vol. 14, N. 2, 308-318.

Krasitskii, V.P. On reduced equations in the Hamiltonian theory of weakly nonlinear surface waves. J. Fluid Mech., 1994, vol. 272, 1 - 30.

Kurkin, A., and Pelinovsky, E. Focusing of edge waves above sloping beach. European Journal of Mechanics - B/Fluid, 2002, vol. 21, N. 5, 561-577.

Lavrenov, I.V. Mathematical modeling of the wind waves in spatially inhomogeneous ocean. Hydrometeozdat. St Petersburg. 1998a.

Lavrenov, I. The wave energy concentration at the Agulhas current of South Africa. Natural Hazards, 1998b, vol. 17, 117 - 127.

Lawton, G. Monsters of the deep (The Perfect Wave). New Scientist. 2001, vol. 170, No. 2297, $28-32$.

Lighthill, M.J. Contribution to the theory of waves in nonlinear dispersive systems. J. Inst. Maths. Application, 1965, vol. 1, 269-306.

Lo, E., and Mei, C.C. A numerical study of water-wave modulation based on a high-order nonlinear Schrodinger equation. J. Fluid Mech., 1985, vol. 150, 395 - 416.

Longuet-Higgins, M. Eulerian and Lagrangian aspects of surface waves. J. Fluid Mech., 1986, vol. $173,683-707$.

Magnusson, A.K., Donelan, M.A., and Drennan, W.M. On estimating extremes in an evolving wave field. Coastal Engineering, 1999, vol. 36, 147 - 163.

Mallory, J.K. Abnormal waves on the south-east of South Africa. Inst. Hydrog. Review, 1974, N $51,89-129$.

Massel, S.R. Ocean surface waves: their physics and prediction. Singapore, World Scientific, 1996.

Matveev, V.B. Positons: slowly decreasing analogues of solitons. Theoretical and Mathematical Physics, 2002, vol. 131, N. 1, 483-497.

Mei, C.C. The applied dynamics of ocean waves. World Sci. 1993.

Miles, J.W. Resonantly interacting solitary waves. J. Fluid Mech., 1977, vol. 79, 171- 179. 
Mori, D., and Yasuda, T. Effects of high-order nonlinear interactions on unidirectional wave trains. Ocean Engineering, 2000, vol. 29, 1233 - 1245.

Mori, N., Liu, P.C., and Yasuda, T. Analysis of freak wave measurements in the Sea of Japan. Ocean Engineering, 2002, vol. 29, 1399-1414.

Olagnon, M. Vagues extrêmes - Vagues scélérates. http://www.ifremer.fr/webcom/molagnon/ipo2000/

Onkuma, K., and Wadati, M. The Kadomtsev - Petviashvili equation, the trace method and the soliton resonance. J. Phys. Soc. Japan, 1983, vol. 52, 749 - 760.

Onorato, M., Osborne, A.R., and Serio, M. Extreme wave events in directional, random oceanic sea states. Phys. Fluids, 2002, vol. 14, N. 4, L25 - L28.

Onorato, M., Osborne, A., Serio, M., and Bertone, S. Freak wave in random oceanic sea states. Phys. Review Letters, 2001, vol. 86, N. 25, 5831-5834.

Onorato, M., Ambrosi, D., Osborne, A.R., and Serio, M. Instability of two interacting, quasimonochromatic waves in shallow water. Physics Fluids, 2003 (submitted).

Osborne, A.R. Solitons in the periodic Korteweg - de Vries equation, the $\Theta$-function representation, and the analysis of nonlinear, stochastic wave trains. Phys. Review E, 1995, vol. 52, N. $1,1105-1122$.

Osborne, A.R., Onorato, M., and Serio, M. The nonlinear dynamics of rogue waves and holes in deep-water gravity wave train. Phys. Letters A, 2000, vol. 275, 386-393.

Osborne, A.R., Serio, M., Bergamasco, L., and Cavaleri, L. Solitons, cnoidal waves and nonlinear interactions in shallow-water ocean surface waves. Physica D, 1998, vol. 123, 64 - 81.

Pelinovsky, E. Hydrodynamics of tsunami waves. Applied Physics Institute Press. Nizhny Novgorod. 1996.

Pelinovsky, E., Talipova, T., and Kharif, C. Nonlinear dispersive mechanism of the freak wave formation in shallow water. Physica D, 2000, vol. 147, N. 1-2, 83-94.

Pelinovsky, E., Kharif, C., Talipova, T., and Slunyaev, A. Nonlinear Wave Focusing as a Mechanism of the Freak Wave Generation in the Ocean. "Rogue Waves 2000” (Brest, France, 2000). Eds: M. Olagnon and G.A.Athanassoulis. Ifremer, 2001a, 193 -204.

Pelinovsky, E., Talipova, T., Kurkin, A., and Kharif, Ch. Nonlinear mechanism of the tsunami wave generation by atmospheric disturbances. Natural Hazards and Earth System Sciences, 2001b, vol. 1, N. 4, 243-250.

Peregrine, D.H. Interaction of water waves and currents. Advanced Applied Mech., 1976, vol. 16, 9-117.

Peregrine, D.H. Wave jumps and caustics in the propagation of finite-amplitude water waves. $J$. Fluid Mech., 1983a, vol. 136, 435 - 452. 
Peregrine, D.H. Water waves, nonlinear Schrodinger equations and their solutions. J. Austral. Math. Soc., ser. B, 1983b, vol. 25, 16-43.

Peregrine, D.H., and Smith, R. Nonlinear effects upon waves near caustics. Phil. Trans. Royal Soc. London, 1979, vol. A292, $341-370$.

Peterson, P., Soomere, T., Engelbrecht, J., and van Groesen, E. Soliton interaction as a possible model for extreme waves. Nonlinear Processes in Geophysics, 2003 (submitted).

Phillips, O.M., Gu, D., and Donelan. M. Expected structure of extreme waves in a Gaussian sea. Pt. 1. Theory and SWADE buoy measurements. J. Phys. Oceanography, 1993, vol. 23, 9921000 .

Sand, S.E., Hansen, N.E., Klinting, P., Gudmestad, O.T., and Sterndorff, M.J. Freak wave kinematics. In: Torum, A., Gudmestad, O.T. (Eds.), Water wave kinematics. Kluwer, Dordrecht, $1990,535-549$.

Satsuma, J. N-soliton solution of the two-dimensional Korteweg - de Vries equation. J. Phys. Soc. Japan, 1976, vol. 40, 286 - 290.

Satsuma J., and N. Yajima, N. Initial value problems of one-dimensional self-modulation of nonlinear waves in dispersive media, Suppl. Prog. Theor. Phys., 1974, vol. 55, 284--306.

Skandrani, C. Contribution à l'étude de la dynamique non linéaire des champs de vagues tridimensionnels en profondeur infinie. PhD Thesis. Université de la Méditerranée 9AixMarseille IIO. 1997.

Shemer, L., Kit, E., Jiao, H., and Eitan, O. Experiments on nonlinear wave groups in intermediate water depth. J. Waterway, Port, Coastal and Ocean Eng., 1998, vol. 124, 320- 327.

Shemer, L., Jiao, H.Y., Kit, E., and Agnon, Y. Evolution of a nonlinear wave field along a tank: experiments and numerical simulations based on spatial Zakharov equation. J. Fluid Mech., 3002, vol. 427, 107 - 129.

Shyu, J.-H., and Tung, C.C. Reflection of oblique waves by currents: analytical solutions and their application to numerical computations. J. Fluid Mech., 1999, vol. 396, 143 - 182.

Slunyaev, A., Kharif, C., Pelinovsky, E., and Talipova, T. Nonlinear wave focusing on water of finite depth. Physica D, 2002, vol. 173. N. 1-2, p. 77-96.

Smith, R. Giant waves. J. Fluid Mech., 1976, vol. 77, 417 - 431.

Smith, S.F., and Swan, C. Extreme two-dimensional water waves: an assessment of potential design solutions. Ocean Engineering, 2002, vol. 29, 387 - 416.

Stansberg, C.T. Random waves in the laboratory - what is expected for the extremes? "Rogue Waves 2000” (Brest, France, 2000). Eds: M. Olagnon and G.A.Athanassoulis. Ifremer, 2001, $289-301$. 
Stiassnie, M., and Shemer, L. On modifications of the Zakharov equation for surface gravity waves. J. Fluid Mech., 1984, vol. 143, 47 - 67.

Trulsen, K. Simulating the spatial evolution of a measured time series of a freak wave. "Rogue Waves 2000” (Brest, France, 2000). Eds: M. Olagnon and G.A.Athanassoulis. Ifremer, 2001, $265-274$.

Trulsen, K., and Dysthe, K.B. A modified nonlinear Schrodinger equation for broader bandwidth gravity waves on deep water. Wave Motion, 1996, vol. 24, 281 - 289.

Trulsen, K., Kliakhandler, I., Dysthe, K.B., and Velarde, M.G. On weakly nonlinear modulation of waves on deep water. Phys. Fluids, 2000, vol. 10, $2432-2437$.

White, B.S., and Fornberg, B. On the chance of freak waves at the sea. J. Fluid Mech., 1998, vol. $255,113-138$.

Whitham G.B. Linear and Nonlinear waves. Wiley, 1974.

Xie, Y. Exact solutions for stochastic KdV equations. Phys. Letters A, 2003, vol. 310, 161 - 167.

Zakharov, V.E. Stability of periodic waves of finite amplitude on the surface of deep water. $J$. Appl. Mech. Tech. Phys., 1968, vol. 9, 190 - 194.

Zakharov, V.E., Dyachenko, A.I., and Vasilyev, O. New method for numerical simulation of nonstationary potential flow of incompressible fluid with a free surface. European J. Mech. B/Fluids, 2002, vol. 21, $283-291$. 